\title{
Metamaterials for Wireless Communications, Radiofrequency Identification, and Sensors
}

\author{
Ferran Martín \\ GEMMA/CIMITEC, Departament d'Enginyeria Electrònica, Universitat Autònoma de Barcelona, \\ Bellaterra, 08193 Barcelona, Spain \\ Correspondence should be addressed to Ferran Martín, ferran.martin@uab.es
}

Received 16 September 2012; Accepted 7 November 2012

Academic Editors: H.-T. Chen and W.-C. Liu

Copyright ( $\odot 2012$ Ferran Martín. This is an open access article distributed under the Creative Commons Attribution License, which permits unrestricted use, distribution, and reproduction in any medium, provided the original work is properly cited.

This paper is focused on the application of concepts derived from metamaterials to the development of novel devices, circuits, and antennas of interest in wireless communications, radiofrequency identification (RFID), and sensors. Specifically, it is shown that artificial transmission lines based (or inspired) on metamaterials exhibit interesting properties, useful for the implementation of high-performance and compact devices as well as novel functional devices. Thanks to the presence of reactive loading elements in such artificial lines, the main line parameters, that is, the characteristic impedance and the phase constant, can be engineered. This has opened new paths for RF and microwave circuit and antenna design on the basis of impedance and dispersion engineering.

\section{Introduction}

With the beginning of the century and the millennium, a new research field appeared in the scene of Science and Technology: Metamaterials. Indeed, the number of journal and conference papers, special sessions in conferences, and research and development projects and contracts related to metamaterials has experienced an exponential growth since 2000. Metamaterials can be considered a transversal topic, involving many different disciplines and fields, such as acoustics, electromagnetism, RF and microwave engineering, millimeter wave and $\mathrm{THz}$ technology, microand nanotechnology, photonics and optics, and medical engineering, among others. During the last years, several books devoted to metamaterials have been published by active researches in the field [1-9]. Such books cover different aspects of metamaterial science and technology, including theory, technology, and applications. In this paper, the focus is on the applications of metamaterial concepts to the design of RF/microwave devices for wireless applications, radiofrequency identification (RFID), and sensors. Therefore, we recommend the reader the books $[1-3,6,8]$ for an indepth treatment of some of the concepts and applications included in this paper, since these books are mainly devoted to electromagnetic metamaterials.
Metamaterials are artificial materials, made of periodic inclusions (or "atoms") of conventional materials (metals and dielectrics) with controllable electromagnetic (or acoustic, or optical) properties. In these artificial materials, the period is much smaller than the guided wavelength; therefore, the structure behaves as a homogeneous (continuous) medium, exhibiting effective medium properties. Such properties can be controlled by properly engineering or structuring the material, and they can be substantially different than those of their constitutive "atoms." For instance, it was experimentally demonstrated in [10] that a prism made of metallic strips and split ring resonators (SRRs) [11] etched on dielectrics slabs exhibits a negative refractive index in a certain frequency band. More recently, the potential of metamaterials was demonstrated for the implementation of invisible cloaks, and an implementation at microwave frequencies was reported in 2006 [12].

Among the achievable effective medium properties of metamaterials, negative refraction was the most exotic one during several years (the implementation of invisible cloaks, however, is now the battle horse in metamaterial research). Negative refraction can be achieved in a medium exhibiting negative effective permittivity and permeability simultaneously. Indeed, such artificial media with negative 


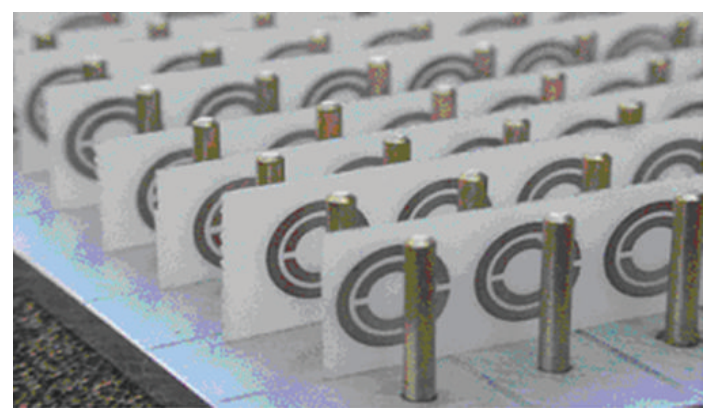

(a)

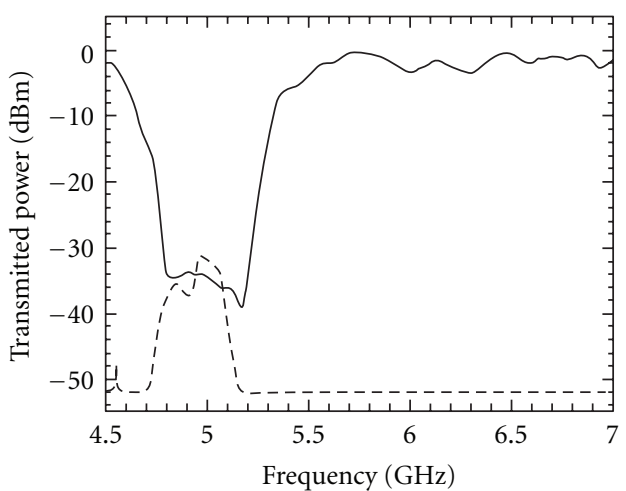

(b)

FIGURE 1: First LH metamaterial fabricated from a periodic array of metallic posts and SRRs, and frequency response (dashed line). The solid line is the measured frequency response of the structure without metallic posts. From [13]; copyright (C) 2000 APS, reprinted with permission.

permittivity and permeability not only exhibit negative refraction, but also backward waves, inverse Doppler Effect, backward Cerenkov radiation, and superresolution (many papers on this topic have been generated after the seminal paper by Pendry et al. [20]). Such properties were already predicted by Veselago in 1968 [21], but it was not until the past decade, after the "big bang" of metamaterials in 2000, that such properties were experimentally demonstrated. The first reported bulk structure exhibiting negative permeability and permittivity was published in 2000 [13] by the Group of Smith (Figure 1). The structure consisted of an array of metallic posts and SRRs. The array of metallic posts behaves as an effective medium with negative permittivity up to a frequency (plasma frequency) that depends on the posts radius and distance between posts; the SRRs exhibit a negative effective permeability in a narrow band above their resonance frequency. Thus, by designing the SRRs in order to present their first resonance in the negative permittivity region of the posts, the combined structure of posts and SRRs exhibits negative constitutive parameters in a certain narrow band. However, the structure is highly anisotropic, since it is necessary to illuminate it with the electric field parallel to the posts and the magnetic field axial to the SRRs. From Maxwell equations, it can be easily demonstrated that a structure with negative permittivity and permeability supports backward waves [6]. Namely, in such media, the phase velocity and the group velocity are antiparallel. The wave vector, the electric field intensity vector, and the magnetic field vector form a left-handed triplet (contrary to conventional materials with positive constitutive parameters), and, for this reason, such materials have been called left-handed (LH) materials.

In $\mathrm{RF}$ and microwave engineering, the constitutive building blocks are transmission lines and stubs. As long as metamaterials exhibit properties beyond those achievable with conventional materials, it is possible to load a transmission line with reactive elements in order to obtain properties not present in conventional lines. Such lines are called metamaterial transmission lines and are the object of the next section. In Section 3, various applications of metamaterial transmission lines for wireless communications are presented, including wide band filters and multiband components. Section 4 is devoted to the design of UHF-RFID chip-based tags designed by using metamaterial concepts, and Section 5 is focused on the application of metamaterial transmission lines for the design of sensors based on the symmetry properties of metamaterial resonators. Finally, the main conclusions are highlighted in Section 6.

\section{Metamaterial Transmission Lines}

Metamaterial transmission lines are artificial lines consisting of a host line (microstrip, coplanar waveguide-CPW-, etc., see Figure 2) loaded with reactive elements (inductances, capacitances, or resonators). Thanks to the presence of these reactive elements, the number of line parameters increases (more degrees of freedom appear as compared to conventional lines), and it is possible to engineer (or tailor) the phase constant and the characteristic impedance of the line. Obviously, the characteristic impedance of conventional transmission lines can also be controlled by the lateral geometry of the line [25-28], but the achievable values are limited by technological constraints and spurious responses. That is, high impedance values require very narrow lines, the limitation being the minimum strip and/or slot width achievable by the available fabrication technology. For low impedance transmission lines, the line width must be necessarily high, and this may cause lateral resonances to appear [29].

Concerning the phase constant, the limitations of conventional lines are even more restrictive. The phase constant in a lossless transmission line is given by

$$
\beta=\omega \sqrt{L^{\prime} C^{\prime}},
$$

where $L^{\prime}$ and $C^{\prime}$ are the per unit length inductance and capacitance of the line and $\omega$ is the angular frequency. This expression can be rewritten in terms of the phase velocity, $v_{p}$, as follows:

$$
\beta=\frac{\omega}{v_{p}}=\frac{\omega}{c} \sqrt{\varepsilon_{r e}}
$$



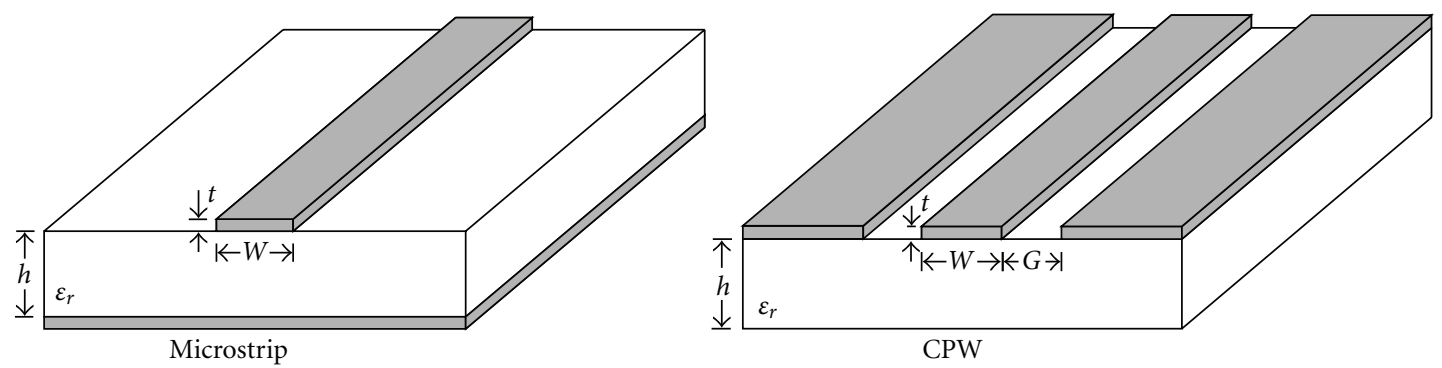

Figure 2: Perspective three-dimensional view of the indicated planar transmission lines, and relevant geometry parameters. These conventional planar transmission lines are the typical host lines used for the implementation of metamaterial transmission lines, as will be later shown. In the microstrip line, the backside metal layer is the ground plane, whereas in CPW technology the ground planes are at the same metal level than the conductor (central) strip.

where $c$ is the speed of light in vacuum and $\varepsilon_{r e}$ is the effective dielectric constant of the line. In closed lines (stripline, for instance-not shown) $\varepsilon_{r e}=\varepsilon_{r}$, the dielectric constant of the substrate; however, in open lines such as microstrip lines or CPWs, where pure transverse electromagnetic (TEM) modes are not supported, the electric field lines are partially present on top of the substrate (air), the effective dielectric constant decreases, and therefore the phase velocity increases. According to (2), conventional lines are (ideally) nondispersive (the phase constant is proportional to frequency) and the group velocity, given by

$$
v_{g}=\left(\frac{d \beta}{d \omega}\right)^{-1}=\frac{1}{\sqrt{L^{\prime} C^{\prime}}}
$$

is identical to the phase velocity. Typically, in microwave applications, transmission lines and stubs of certain electrical length $(\beta d)$ are required. Many microwave circuits are based on $90^{\circ}$ and $180^{\circ}$ lines, which correspond to quarter- and half-wavelength transmission lines, respectively, since the wavelength is given by

$$
\lambda=\frac{2 \pi}{\beta} .
$$

In view of expression (2), it is clear that to reduce the physical length of the line, $d$, it is necessary to reduce the phase velocity as much as possible. This increases $\beta$ and therefore decreases $d$ for a given (required) electrical length at the operating frequency (which is the typical design parameter). According to (2), size reduction can be achieved by increasing the effective dielectric constant. As long as choosing the substrate is a possible option, high dielectric constant substrates are the preferred solution for size reduction. However, this is not always possible; therefore, the phase velocity is not actually a design parameter. In other words, in conventional lines, the impedance can be tailored within restrictive limits, and dispersion cannot be engineered. This is the main difference as compared to metamaterial transmission lines, where circuit design is based on impedance and dispersion engineering.

One difference between metamaterials and metamaterial transmission lines is that whereas in metamaterials the main aim is the controllability of the constitutive parameters (permeability and permittivity), the main objective in metamaterial transmission lines is to control the line impedance and phase constant (or dispersion). Periodicity and homogeneity are important in metamaterials, but such aspects are not relevant for $\mathrm{RF} /$ microwave circuit design. Nevertheless, if metamaterial transmission lines are made by cascading several unit cells and the homogeneity conditions are fulfilled, such artificial lines can be considered onedimensional metamaterials and effective constitutive parameters can be defined, as will be later shown.

2.1. The Backward Transmission Line. Attracted by the potential of LH materials, several groups focused their efforts on the implementation of transmission lines exhibiting LH wave propagation [30-32]. Indeed, the concept of backward waves to describe propagating waves with antiparallel phase and group velocities is not new (several textbooks have dealt with and described this subject $[33,34])$. Backward waves can be generated by means of a ladder network consisting of a cascade of shunt connected inductors and series capacitors (Figure 3). This network is the dual counterpart of the equivalent circuit model of a conventional lossless planar transmission line with forward (as opposed to backward) wave propagation (see also Figure 3). The analysis of the propagation characteristics of the structures shown in Figure 3 can be realized from the theory of periodic structures, where it is assumed that the structures are either infinite or matched to the ports. From this analysis, the phase constant and the characteristic impedance are given by [25]

$$
\begin{gathered}
\cos \beta l=1+\frac{Z_{s}(\omega)}{Z_{p}(\omega)}, \\
Z_{B}=\sqrt{Z_{s}(\omega)\left[Z_{s}(\omega)+2 Z_{p}(\omega)\right]},
\end{gathered}
$$

where $Z_{s}(\omega)$ and $Z_{p}(\omega)$ are the series and shunt impedances of the basic cell of the structures described by the T-circuit model, and $l$ is the period. If the $\pi$-circuit model of the unit cell is considered, the phase constant is also given by expression (5), but the characteristic impedance is given by

$$
Z_{B}(\omega)=\sqrt{\frac{Z_{s}(\omega) Z_{p}(\omega) / 2}{1+Z_{s}(\omega) / 2 Z_{p}(\omega)}} .
$$




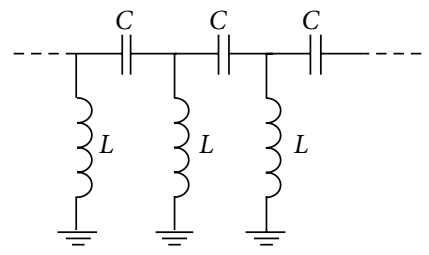

(a)

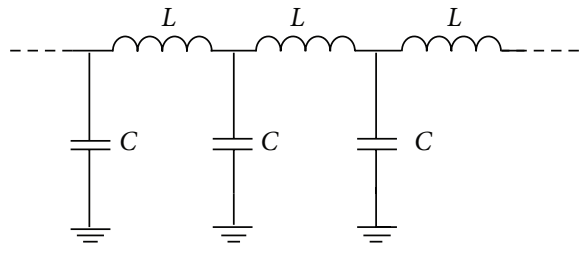

(b)

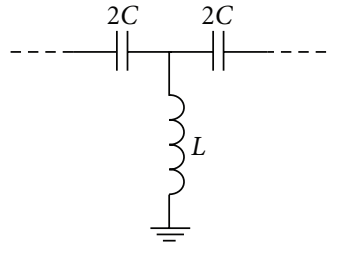

(c)

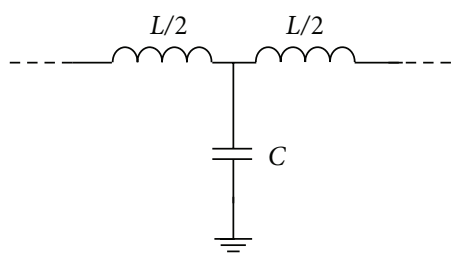

(d)

FIGURE 3: Equivalent circuit model of backward (a) and forward (b) transmission lines. The T-circuit models of the basic cell structures are also indicated in (c) and (d).

Application of expressions (5) and (6) to the unit cell (Tcircuit) of the backward line gives

$$
\begin{aligned}
& \cos \beta_{L} l=1-\frac{1}{2 L C \omega^{2}}, \\
& Z_{B L}=\sqrt{\frac{L}{C}\left(1-\frac{\omega_{c L}^{2}}{\omega^{2}}\right)},
\end{aligned}
$$

whereas for the forward transmission line model we obtain

$$
\begin{gathered}
\cos \beta_{R} l=1-\frac{L C}{2} \omega^{2}, \\
Z_{B R}=\sqrt{\frac{L}{C}\left(1-\frac{\omega^{2}}{\omega_{c R}^{2}}\right)},
\end{gathered}
$$

where the subscripts $R$ and $L$ are used to distinguish between the $\mathrm{LH}$ and the right-handed $(\mathrm{RH})$ structures, and $\omega_{c R}=$ $2 /(L C)^{1 / 2}$ and $\omega_{c L}=1 / 2(L C)^{1 / 2}$ are angular cut-off frequencies. The dispersion diagram and the dependence of $Z_{B}$ on frequency are depicted in Figure 4. Transmission is limited to those frequency intervals that make the phase constant and the characteristic impedance to be real numbers.

It is worth mentioning that frequency dispersion is present in both structures. Even though the circuit of Figure 3(b) models an ideal lossless forward transmission line, where dispersion is absent, actually this circuit is only valid for frequencies satisfying $\omega \ll \omega_{c R}$, that is, in the long wavelength limit (corresponding to those frequencies where wavelength for guided waves satisfies $\lambda \gg l$ ). To correctly model an ideal lossless transmission line at higher frequencies, we simply need to reduce the period of the structure, and accordingly the per section inductance and capacitance of the line, $L$ and $C$, with the result of a higher cut-off frequency. Thus, the circuit of Figure 3(b) can properly describe ideal transmission lines without dispersion. To this end we simply need to select the period such that the long wavelength limit approximation holds. Under this approximation, expressions (10) and (11) lead us to the following well known expressions:

$$
\begin{gathered}
\beta_{R}=\omega \sqrt{L^{\prime} C^{\prime}}, \\
Z_{B R}=\sqrt{\frac{L^{\prime}}{C^{\prime}}} \equiv Z_{l w},
\end{gathered}
$$

where $L^{\prime}$ and $C^{\prime}$ are the per unit length inductance and capacitance of the transmission line (in expression (13), $L^{\prime}$ and $C^{\prime}$ can be replaced by $L$ and $C$ with no effect). From (12), we can obtain the phase and group velocities of the forward transmission line. These velocities are given by

$$
\begin{gathered}
v_{p R}=\frac{\omega}{\beta_{R}}=\frac{1}{\sqrt{L^{\prime} C^{\prime}}}=\frac{l}{\sqrt{L C}} \\
v_{g R}=\left(\frac{\partial \beta_{R}}{\partial \omega}\right)^{-1}=v_{p R}
\end{gathered}
$$

and they are both positive and constant.

Conversely, the backward wave structure of Figure 3(a) is dispersive even in the long wavelength limit. In order to identify this structure as an effective propagating medium (i.e., one-dimensional metamaterial), operation under this approximation is required. However, regardless of the operating frequency within the transmission band, the structure supports backward waves and, for this reason, it is an LH transmission line. In order to properly identify effective constitutive parameters, $\mu_{\text {eff }}$ and $\varepsilon_{\text {eff }}$, the long wavelength approximation is necessary. However, many circuit applications of these LH lines are based on left handedness, rather than on the effective medium properties, and operation under this approximation is not a due. Nevertheless, for coherence and simplicity, the phase constant, the characteristic impedance, and the phase and group velocities are derived under the long 


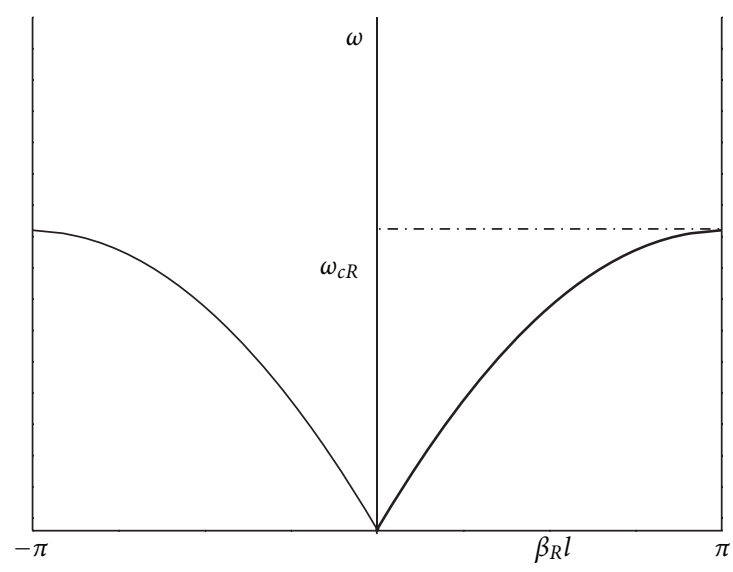

(a)

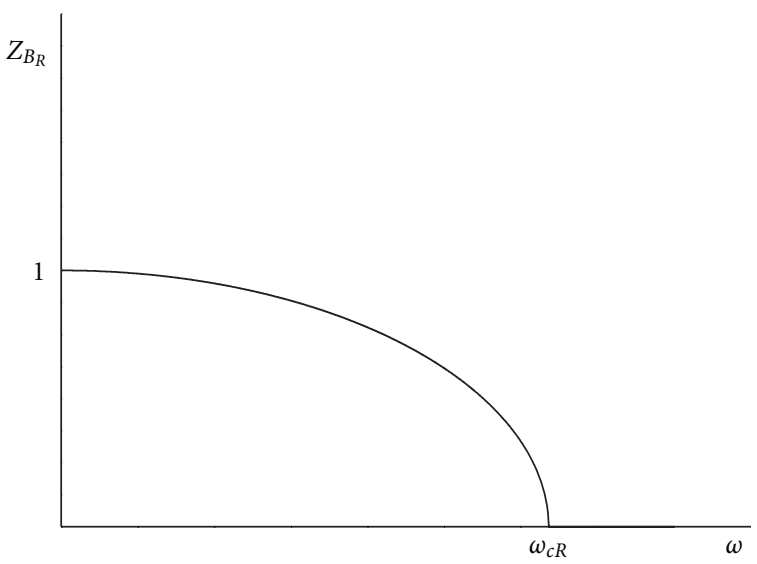

(c)

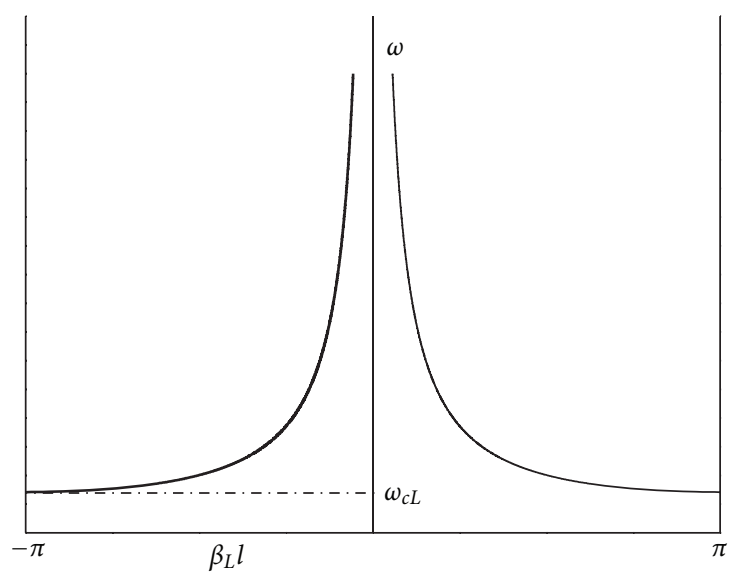

(b)

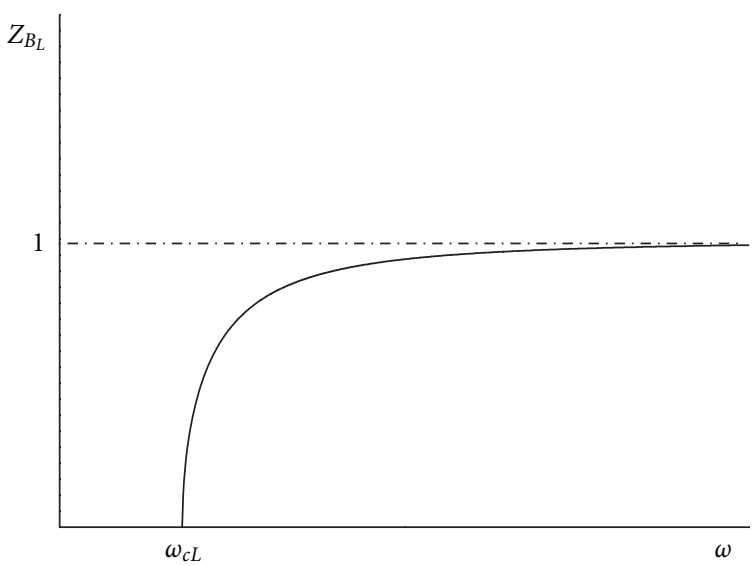

(d)

FIGURE 4: Typical dispersion diagram of a forward (a) and backward (b) transmission line model. The dependence of the normalized $\left(Z_{B} / Z_{l w}\right)$ Bloch impedance with frequency is shown in (c) and (d) for the forward and backward lines, respectively. $Z_{l w}$ is the maximum value of the impedance. Notice that for the backward transmission line the group velocity tends to infinity as frequency increases. Actually, the model is not valid at high frequencies since this implementation requires a host line that introduces parasitic elements to the model.

wavelength limit $\left(\omega \gg \omega_{c L}\right)$. The following results are obtained:

$$
\begin{gathered}
\beta_{L} l=-\frac{1}{\omega \sqrt{L C}}, \\
Z_{B L}=\sqrt{\frac{L}{C} \equiv Z_{l w},} \\
v_{p L}=\frac{\omega}{\beta_{L}}=-\omega^{2} l \sqrt{L C}<0, \\
v_{g L}=\left(\frac{\partial \beta_{L}}{\partial \omega}\right)^{-1}=+\omega^{2} l \sqrt{L C}>0
\end{gathered}
$$

and the phase and group velocities have opposite signs. From a mathematical point of view, in the dispersion diagrams for the forward and backward lines depicted in Figure 4, and obtained from expressions (8) and (10), the sign of $\beta$ can be either positive or negative. This ambiguity comes from the two possible directions of energy flow, namely, from left to right or vice versa. If we adopt the usual convention of energy flow from left to right, then the sign of the phase constant is determined by choosing that portion of the curves that provide a positive group velocity (bold lines in Figures 4(a) and 4(b)). From this, it is clear that for the forward transmission line, $\beta$ and $v_{p}$ are both positive, whereas these magnitudes are negative for the backward transmission line. In both cases, $v_{g}$ is positive as one expects on account of the codirectionality between power flow and group velocity.

In the backward and forward transmission lines depicted in Figure 3, it is possible to identify effective constitutive parameters. To this end, we should take into account that transverse electromagnetic (TEM) mode propagation in planar transmission media and plane wave propagation in isotropic and homogeneous dielectrics are described by identical equations (telegraphist's equation) provided the following mapping holds:

$$
\begin{aligned}
& Z_{s}^{\prime}(\omega)=j \omega \mu_{\mathrm{eff}}, \\
& Y_{p}^{\prime}(\omega)=j \omega \varepsilon_{\mathrm{eff}},
\end{aligned}
$$




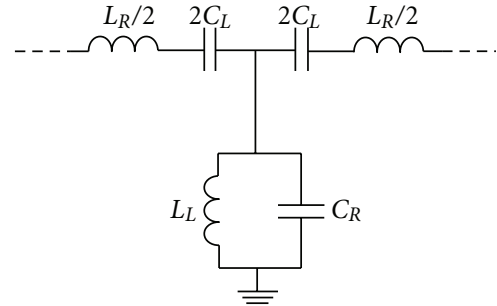

Figure 5: Equivalent circuit model (basic cell) of the CRLH transmission line.

where $Z_{s}^{\prime}$ and $Y_{p}^{\prime}$ are the series impedance and shunt admittance per unit length. Thus, for the forward line the effective permittivity and permeability are constant and they are given by

$$
\begin{aligned}
& \varepsilon_{\text {eff }}=\frac{C}{l}, \\
& \mu_{\text {eff }}=\frac{L}{l},
\end{aligned}
$$

whereas for the backward transmission line, the constitutive parameters are

$$
\begin{aligned}
& \varepsilon_{\text {eff }}=-\frac{1}{\omega^{2} L l}, \\
& \mu_{\text {eff }}=-\frac{1}{\omega^{2} C l}
\end{aligned}
$$

and they are both negative, a sufficient condition to obtain LH wave propagation.

2.2. Composite Right/Left-Handed (CRLH) Transmission Lines. From a practical viewpoint, in order to implement a LH line, a host line is required. The host line introduces parasitic elements, that is, the line inductance and the line capacitance. Therefore, the T-circuit model of the unit cell of these lines is that depicted in Figure 5, where $C_{R}$ and $L_{R}$ are the line parameters, and the $C_{L}$ and $L_{L}$ are the loading elements of the line.

The dispersion relation and the characteristic impedance, inferred from expressions (5) and (6), are

$$
\begin{gathered}
\cos \beta l=1-\frac{\omega^{2}}{2 \omega_{R}^{2}}\left(1-\frac{\omega_{s}^{2}}{\omega^{2}}\right)\left(1-\frac{\omega_{p}^{2}}{\omega^{2}}\right), \\
Z_{B}=\sqrt{\frac{L_{R}}{C_{R}} \frac{\left(1-\omega_{s}^{2} / \omega^{2}\right)}{\left(1-\omega_{p}^{2} / \omega^{2}\right)}-\frac{L_{R}^{2} \omega^{2}}{4}\left(1-\frac{\omega_{s}^{2}}{\omega^{2}}\right)^{2}},
\end{gathered}
$$

where the following variables

$$
\begin{aligned}
& \omega_{R}=\frac{1}{\sqrt{L_{R} C_{R}}}, \\
& \omega_{L}=\frac{1}{\sqrt{L_{L} C_{L}}},
\end{aligned}
$$

and the series and shunt resonance frequencies

$$
\begin{aligned}
& \omega_{s}=\frac{1}{\sqrt{L_{R} C_{L}}}, \\
& \omega_{p}=\frac{1}{\sqrt{L_{L} C_{R}}},
\end{aligned}
$$

have been introduced to simplify the mathematical formulas. Expressions (19) and (20) are depicted in Figure 6. Two propagating regions, separated by a gap at the spectral origin, can be distinguished. In the lowest frequency region the parameters of the backward transmission line, $C_{L}$ and $L_{L}$, are dominant and wave propagation is backward. This situation is reversed above the stop band, where the parasitic reactances of the host line make the structure to behave as a right-handed line. Clearly, the structure exhibits a composite right/left-handed (CRLH) behaviour and for this reason those structures modelled by the T-circuit of Figure 5 (unit cell) are called CRLH lines [35].The gap limits are given by the frequencies satisfying

$$
\begin{aligned}
& \omega_{G 1}=\min \left(\omega_{s}, \omega_{p}\right), \\
& \omega_{G 2}=\max \left(\omega_{s}, \omega_{p}\right) .
\end{aligned}
$$

In the long wavelength limit, expression (19) rewrites as

$$
\beta=\frac{s(\omega)}{l} \sqrt{\frac{\omega^{2}}{\omega_{R}^{2}}\left(1-\frac{\omega_{s}^{2}}{\omega^{2}}\right)\left(1-\frac{\omega_{p}^{2}}{\omega^{2}}\right)},
$$

where $s(\omega)$ is the following sign function:

$$
s(\omega)= \begin{cases}-1 & \text { if } \omega<\min \left(\omega_{s}, \omega_{p}\right), \\ +1 & \text { if } \omega>\max \left(\omega_{s}, \omega_{p}\right) .\end{cases}
$$

From the phase constant (24), the phase and group velocities can be easily inferred, these velocities being of opposite sign in the left-handed band and both being positive in the right handed band. With regard to the constitutive parameters for the CRLH transmission line, they can be inferred as previously indicated, that is,

$$
\begin{aligned}
& \varepsilon_{\text {eff }}=\frac{C_{R}}{l}-\frac{1}{\omega^{2} L_{L} l}, \\
& \mu_{\text {eff }}=\frac{L_{R}}{l}-\frac{1}{\omega^{2} C_{L} l}
\end{aligned}
$$

and they can be positive or negative, depending on the frequency range.

One particular case of interest of CRLH lines is the one with identical series and shunt resonance frequencies, $\omega_{s}=$ $\omega_{p}=\omega_{o}[2]$ (balanced CRLH transmission line). In this case, the gap collapses and the transition between the LH and the $\mathrm{RH}$ band is continuous (both regions are separated by the socalled transition frequency, $\left.\omega_{o}\right)$. The dispersion relation and the characteristic impedance for a typical balanced CRLH line are depicted in Figure 7. The impedance reaches its maximum at $\omega_{o}$, where

$$
Z_{B}=\sqrt{\frac{L_{R}}{C_{R}}}=\sqrt{\frac{L_{L}}{C_{L}}}
$$




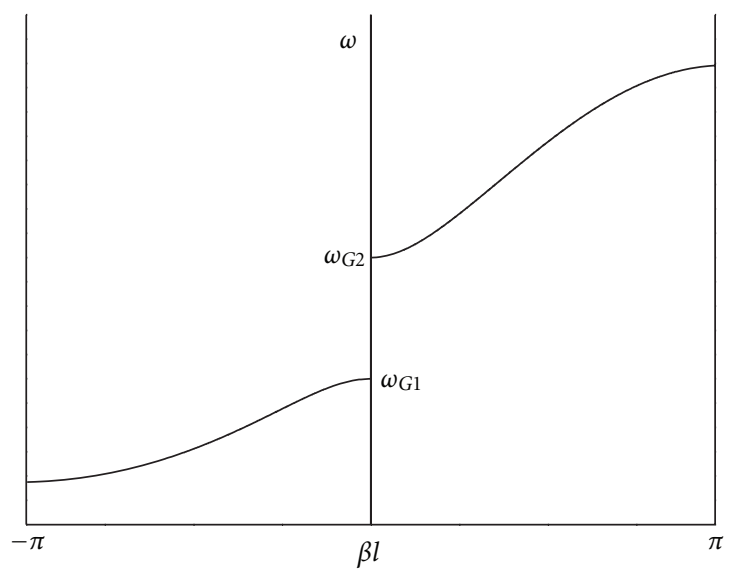

(a)

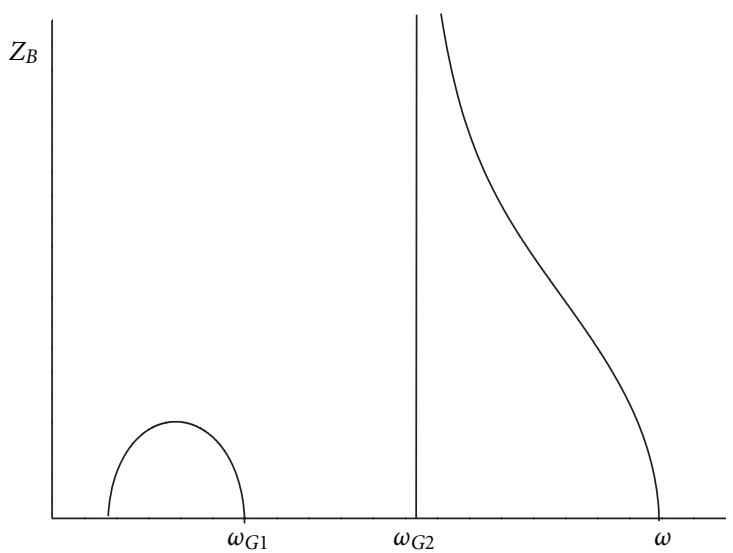

(b)

FIGURE 6: Typicaldispersion diagram (a) and variation of characteristic impedance with frequency (b) in a CRLH transmission line model. In this example, $\omega_{s}<\omega_{p}$.

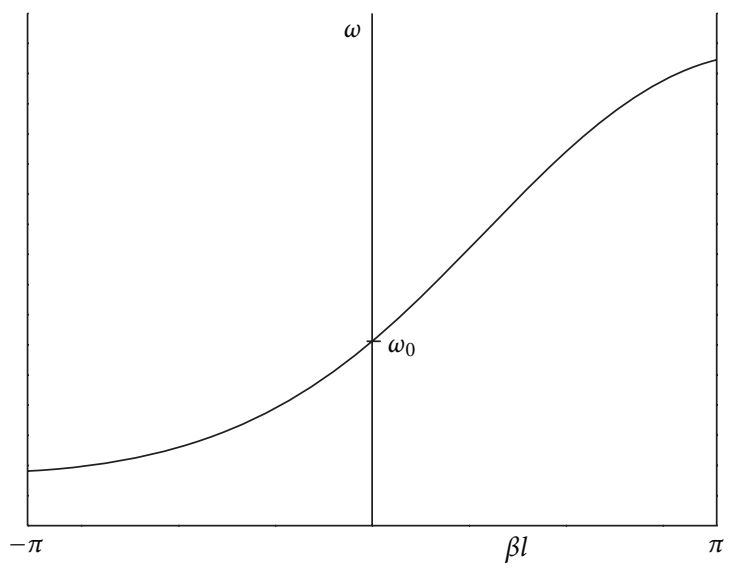

(a)

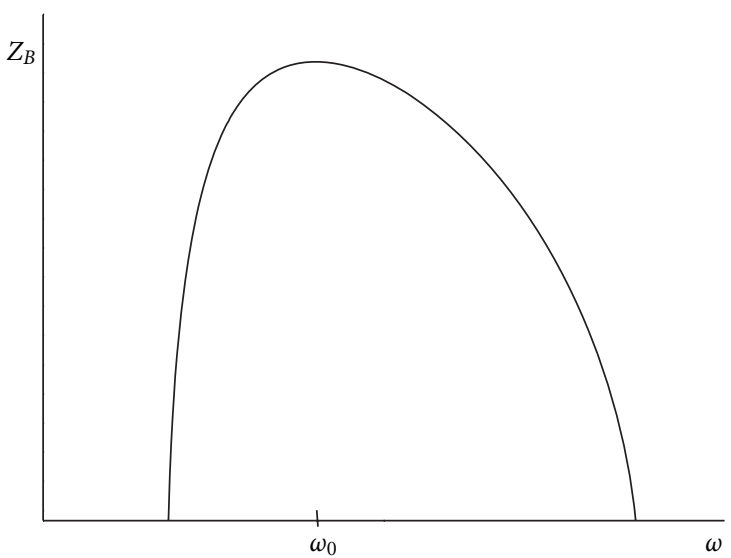

(b)

FIGURE 7: Typical dispersion diagram (a) and variation of characteristic impedance with frequency (b) in a balanced CRLH transmission line.

and the characteristic impedance decreases as frequency increases or decreases from $\omega_{0}$.

The balanced case exhibits another important difference as compared to the unbalanced CRLH transmission line. Namely, at the transition frequency, where the phase velocity exhibits a pole, the phase shift is zero, and the group velocity is different than zero. In other words, at this frequency, wave propagation is possible $\left(v_{g} \neq 0\right)$ with $\beta=0$. The phase origin of the balanced CRLH line takes place thus at $\omega_{o}$. When frequency is decreased below $\omega_{o}$, the phase becomes positive and it increases progressively; conversely, when frequency increases from $\omega_{o}$, the phase increases in magnitude but with negative sign (as occurs in conventional transmission lines). Concerning the behaviour of wavelength for guided waves, $\lambda$, in the balanced CRLH transmission line, this reaches its maximum (infinity) at $\omega_{o}$, and $\lambda$ decreases as frequency increases or decreases from $\omega_{0}$. Indeed, for the unbalanced case, guided wavelength also increases when frequency approaches the spectral gap. However, at the edges of the gap the group velocity is zero, the line impedance takes extreme values, and signal propagation is not allowed.

2.3. Other CRLH Transmission Lines. Transmission lines exhibiting CRLH behaviour can also be realized by means of the dual network to that shown in Figure 5 [36]. The T-circuit model of this dual-CRLH (D-CRLH) is depicted in Figure 8. As compared to the CRLH transmission line, the D-CRLH line exhibits forward wave propagation at low frequencies and backward wave propagation at high frequencies. The structure exhibits stop band behaviour, and this stop band functionality is preserved even for balanced D-CRLH lines.

Both CRLH and D-CRLH lines exhibit a right-handed and a left-handed bands. However, by increasing the number of reactive elements in the transmission line model, it is 


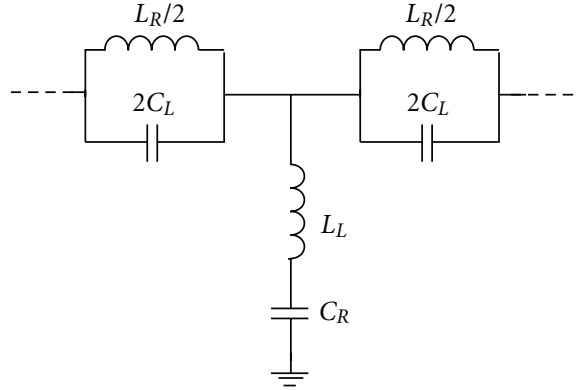

FIGURE 8: Circuit model of the unit cell of a D-CRLH transmission line.

possible to increase the number of forward and backward wave transmission bands. CRLH lines can be labelled by their order, given by the number of allowed bands. Thus, the forward and backward transmission lines of Figures 3(a) and 3 (b) are order- 1 lines, whereas the CRLH and D-CRLH lines of Figures 5 and 8 are order-2 lines. Notice that the order is related to the number of reactive elements of the series or shunt branch in the T-circuit model. The order can be made arbitrarily high by means of the generalized networks shown in Figure 9. The first one (Figure 9(a)) is the generalized model of a CRLH line [37]. This structure exhibits alternate backward and forward bands, the first one being backward. The structure depicted in Figure 9(b) is the dual counterpart of the structure of Figure $9(\mathrm{a})$ and is thus the generalized model of the D-CRLH line. The practical implementation of the circuits of Figure 9 is not straightforward and, typically, order-4 CRLH lines are implemented by means of the model depicted in Figure 10 [38, 39]. Indeed, it was demonstrated in [37] that the model of Figure 10 is equivalent to the model of Figure 9(a); that is, transformation equations between the elements of both models do exist. This can be made extensible to any line order; namely, any CRLH or DCRLH transmission line model can be transformed to those generalized models depicted in Figure 9. In the section devoted to applications, the potentiality of order-4 CRLH transmission lines will be highlighted.

2.4. Implementation of Metamaterial Transmission Lines. There are two main approaches for the implementation of metamaterial transmission lines: (i) the CL-loaded approach, where the host line is loaded with series capacitances and shunt inductances, and (ii) the resonant-type approach, where the line is loaded with electrically small resonators, such as SRRs or other related resonators, plus additional reactive elements. Let us review some practical implementations of these line types, with special emphasis on the resonant-type approach (which represents the main contribution of the author in the field of metamaterials).

2.4.1. CL-Loaded Lines. CL-loaded CRLH transmission lines consist of a host line loaded with series capacitances and shunt inductances. Obviously, the reactive loading can be achieved by means of lumped elements, but this represents extra cost. Therefore, the use of semilumped elements is of the highest interest. CRLH transmission lines in microstrip technology were proposed by Caloz et al. [40]. The reported structure consists of a periodic arrangement of series interdigital capacitors alternated with grounded (through metallic vias) stubs, which act as shunt connected inductors (see Figure 11). The interdigital capacitors are described by the series capacitance $C_{L}$ in the circuit of Figure 5, whereas $L_{L}$ models the grounded stubs. The other reactive elements $C_{R}$ and $L_{R}$ correspond to the line capacitance and inductance, respectively.

In CPW configuration, the shunt inductors can be implemented by means of connecting strips between the central strip and the ground planes. The series capacitors can be implemented through interdigital geometries, or by means of series gaps. The latter are simpler, but the achievable capacitance values are much smaller. The Eleftheriades' Group at the University of Toronto (Canada) presented a realization of a left-handed line fabricated in CPW technology by using series gaps and shunt strips (see Figure 12) [14].

2.4.2. Resonant-Type CRLH Transmission Lines. Resonanttype CRLH transmission lines are based on SRRs and other electrically small resonators. Therefore, let us first dedicate some words to such resonant particles. The topology and the equivalent circuit model of the SRR are depicted in Figure 13(a). It consists of a pair of split rings with the slits etched in opposite sides. This particle can be excited by an axial time-varying magnetic field (it can also be driven by an electric field applied in the plane of the particle [41]). Above resonance, the currents induced by the incident magnetic field generate a magnetic field in opposite direction to the external one, and for this reason an array of SRRs illuminated with the magnetic field axial to the rings behaves as a negative effective permeability medium. Homogeneity can be achieved since SRRs are electrically small resonators. This small electrical size is originated by the coupling between the inner and the outer rings [42]. Thanks to such coupling, the first resonance frequency of the SRR is smaller than the resonance frequencies of the individual rings, whereas the second resonance frequency of the particle lies beyond those of the individual rings. In practice, the coupling between the inner and the outer ring is limited by the slot width between both rings. Such slot widths cannot be smaller than roughly $100 \mu \mathrm{m}$ in standard PCB technology, which means that the electrical size of the particles (diameter) cannot be driven below $\lambda / 10$. However, the electrical size of the SRR can be substantially reduced by etching the rings face-toface in opposite sides of a thin dielectric slab [41]. The capacitance between both rings is broad side, rather than edge side, and the coupling between the inner and the outer ring can be significantly increased by merely using very thin substrates. However, this strategy is not always possible, and an alternative path for size reduction is to use spiral resonators, SRs $[43,44]$.

Since the SRR can be considered to be electrically small, a quasistatic analysis to infer the SRR inductance and capacitance can be applied. Such analysis was carried out 


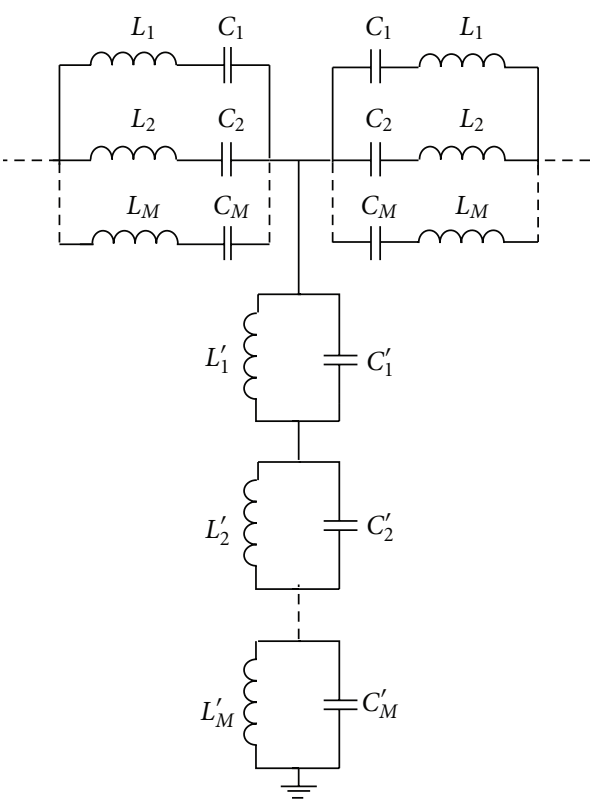

(a)

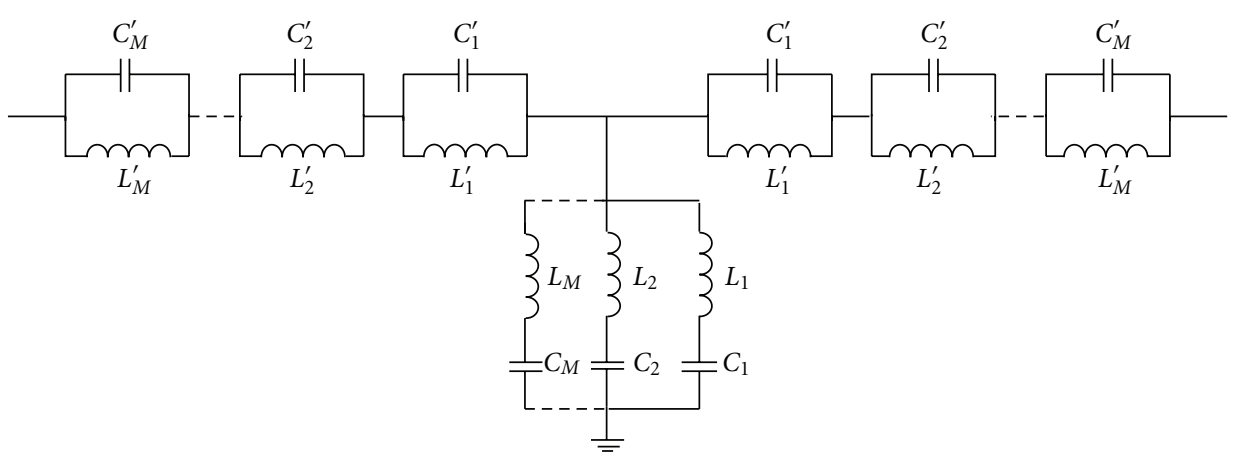

(b)

FIgURe 9: Generalized model of a CRLH (a) and D-CRLH line (b).

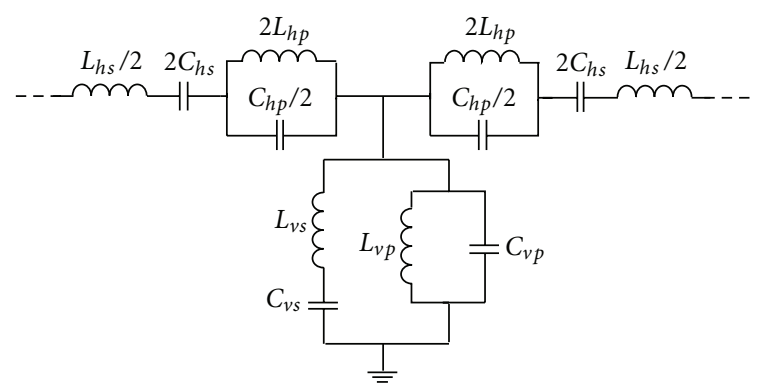

FIGURE 10: Circuit model of an order-4 CRLH line, typically used for practical implementations.

in $[6,45]$. When the SRR is excited by an external timevarying magnetic field directed along the SRR axis, the cuts on each ring force the electric current to flow from one ring to another across the slot between them, taking the form of a strong displacement current. The slot between the rings therefore behaves as a distributed capacitance, and the whole SRR has the equivalent circuit shown in Figure 13(a), where

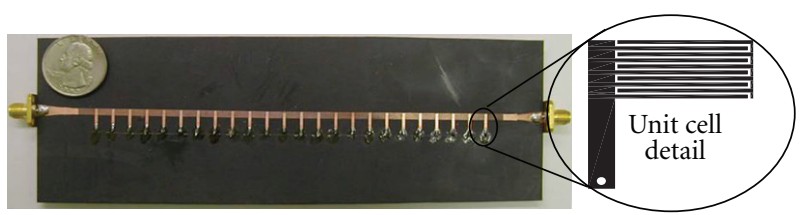

FIgURE 11: Microstrip CRLH transmission line implemented by means of shunt connected grounded stubs and interdigital capacitors. Photo courtesy by C. Caloz.

$L_{s}$ is the SRR self-inductance and $C_{o} / 2$ is the capacitance associated with each SRR half. This capacitance is $C_{o}=$ $2 \pi r_{o} C_{\text {pul }}$, where $r_{o}$ is the mean radius of the SRR, and $C_{\text {pul }}$ is the per unit length capacitance along the slot between the rings. The total capacitance of this circuit is the series connection of the capacitance of both SRR halves, that is, $C_{o} / 4$. Therefore, the resonance frequency $\omega_{o}$ is given by

$$
\omega_{o}=\frac{2}{\sqrt{L_{s} C_{o}}}=\sqrt{\frac{2}{\pi r_{o} L_{s} C_{\mathrm{pul}}}} .
$$




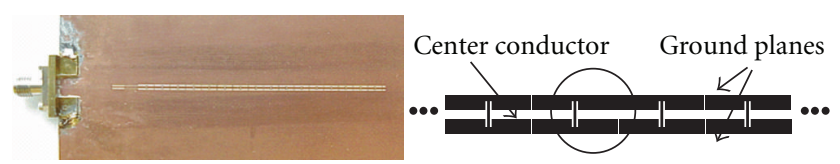

FIGURE 12: CPW CRLH transmission line acting as a leaky wave antenna. From [14]; copyright (C2002 American Institute of Physics, reprinted with permission.

The inductance $L_{s}$ can be modeled as the inductance of an average ring of radius $r_{o}$, that is, the mean radius of the ring, and width $c$. Approximate expressions for $L_{s}$, based on a variational calculation, are provided in [41]. Closed expressions for the per unit length capacitance are given in many microwave textbooks [28], and explicit expressions for $L_{s}$ and $C_{\text {pul }}$ are provided in [6].

The model of Figure 13(a) is valid as long as the perimeter of the ring can be considered small with regard to half wavelength, and the capacitance associated with the cuts on each ring can be neglected. Under such assumptions, the currents on each ring must vanish at the cuts, and the angular dependence of the currents on each ring can be assumed to be linear (so that the total current on both rings is constant). Such assumptions also imply that the voltage across the slots is constant in both SRR halves. A more detailed circuit model, which takes into account the gap capacitance and includes a transmission line model for the slot between the rings, has been reported in [46], but such model converges to the model of Figure 13(a) when the capacitance of the cuts is neglected and the electrical length of the SRR is small [46]. As both approximations are usually fulfilled for any practical SRR design, the more simple model reported above is assumed valid in this paper.

Let us now consider the application of duality to the SRR. If the topology of the SRR is removed from a metallic film, the resulting particle (Figure 13(b)) can be considered the negative image, or the complementary counterpart, of the SRR. For this reason, this particle has been called complementary split ring resonator (CSRR) [47]. It can be easily demonstrated by applying the Babinet principle, that the CSRR can be excited by means of an axial timevarying electric field or by means of a magnetic field applied to the plane of the particle [48]. Under ideal conditions, that is, a perfectly conducting and zero-thickness metallic screen, it can be demonstrated that the resonance frequency of the CSRR is identical to the resonance frequency of the SRR (provided identical dimensions and substrate are considered). Indeed, the capacitance of the CSRR, $C_{c}$, is the capacitance of a disk of radius $r_{o}-c / 2$ surrounded by a ground plane at a distance $c$ of its edge. The inductance is given by the parallel connection of the two inductances of the metallic strips connecting the inner and outer metallic regions of the CSRR. These inductive values are given by $L_{o} / 2$, where $L_{o}=2 \pi r L_{\mathrm{pul}}$, with $L_{\mathrm{pul}}$ being the per unit length inductance of the CPWs connecting the inner disk to ground.

The SRR and the CSRR are closed particles that can be externally driven by a magnetic or by an electric field with the adequate polarization. Let us now consider open resonators inspired on these metamaterial resonant particles. Open particles can be of interest in applications where the resonator must be excited by means of a voltage or current source. Inspired on the topology of the SRR of Figure 13(a), the open split ring resonator (OSRR) was presented in 2004 [49]. This particle can be constructed by means of two concentric rings with cuts in the same position. By elongating the rings, shaping them as a hook (Figure 13(c)), we create the two required terminals for particle excitation. The incident current is transferred from one ring to the other through the distributed edge capacitance between the two rings. According to that, it is clear that the capacitance of this particle is four times larger than the capacitance of the SRR. Since the inductance of the OSRR is identical to the inductance of the SRR, it follows that the resonance frequency of the OSRR is half the resonance frequency of the SRR. In other words, the OSRR is electrically smaller than the SRR by a factor of two. Therefore, OSRRs are very interesting for applications where small series (open) resonators implemented in one metal layer are required. As will be later shown, OSRRs are useful for the implementation of metamaterial-based devices, but these particles have also been applied to the design of band pass filters based on series resonators coupled through impedance inverters [50].

Complementarity can also be applied to the OSRR. The complementary version of the OSRR was called open complementary split ring resonator (OCSRR) [51]. The OCSRR is the complementary (negative) image of the OSRR, but it can also be considered to be the open version of the CSRR. However, the OCSRR must be considered with some caution, since the metallic terminals of the OSRR disappear by applying duality. As shown in Figure 13(d), the OCSRR is an open particle with terminals for external connection (excitation) as indicated in the figure. From one terminal to the other there are two possible current paths: on one hand, the inductive path through the strip between the ring slots; on the other hand, the capacitive path through the slots. Therefore, the OCSRR is an open parallel resonant tank. The capacitance of the particle is the capacitance of the CSRR, $C_{c}$; the inductance is four times larger than the inductance of the CSRR. This means that the OCSRR is electrically smaller than the CSRR by a factor of two. Indeed, the resonance frequency and electrical size of the OSRR and the OCSRR are identical (provided the same dimensions and substrate are considered).

Once the main relevant metamaterial resonators have been considered, let us review the main implementations of CRLH metamaterial transmission lines based on them. CRLH lines can be implemented by loading a host CPW with pairs of SRRs and shunt connected strips, or by loading a microstrip line with CSRRs (etched in the ground plane) and series capacitive gaps. If the host lines are only loaded with SRRs or CSRR, the structures exhibit a stop band behavior that can be attributed to a negative value of the permeability (SRR-loaded lines) or permittivity (CSRR-loaded lines) in a narrow band in the vicinity of particle resonance. These SRR- or CSRR-loaded lines have been applied to the design of stop band filters, or for spurious and harmonic suppression in conventional filters and other microwave devices [52-58]. 


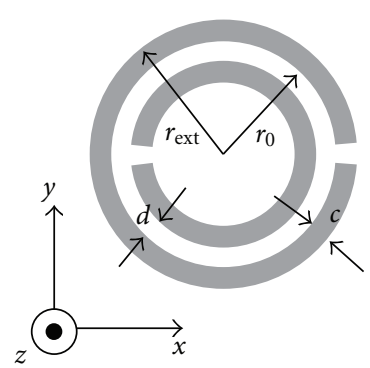

(a)

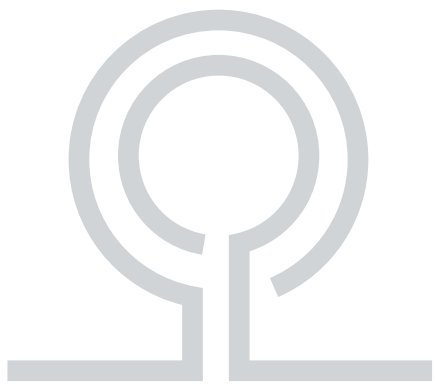

(c)
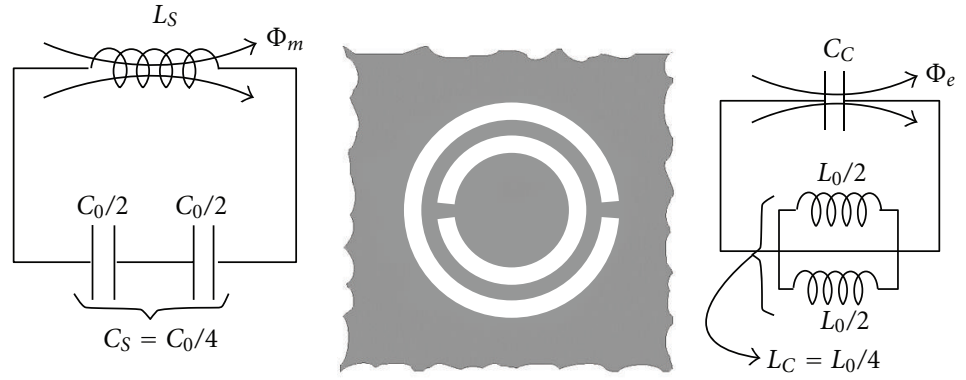

(b)
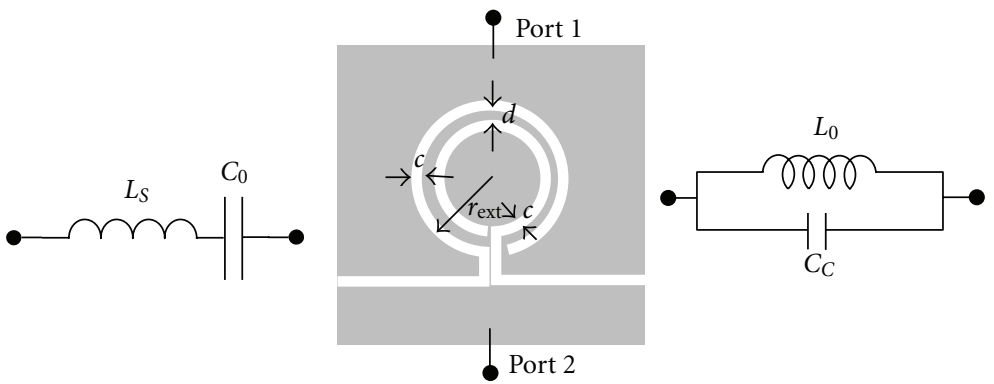

(d)

FiguRE 13: Typical topology and circuit model of several metamaterial resonant particles: SRR (a); CSRR (b); OSRR (c); OCSRR (d).

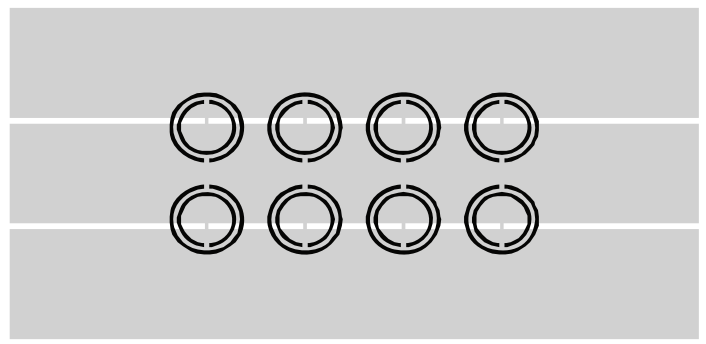

FIGURe 14: Typical layout of a CRLH SRR-based CPW transmission line. The SRRs are etched in the back substrate side.

CRLH lines can also be implemented by loading a host line with OSRR and OCSRR alternating in the line. Let us now review the circuit models of such CRLH transmission lines.

(1) SRR-Based Metamaterial Transmission Lines. By etching SRRs in the slot regions of a CPW transmission line and shunt connected strips between the central strip and the ground planes, a CRLH transmission line results (Figure 14) [59]. The circuit model of these CRLH lines (unit cell) is shown in Figure 15(a), where $L$ and $C$ are the per-section inductance and capacitance of the line, $L_{p}$ models the inductance of the shunt strips, and the SRRs are described by the resonant tank $L_{s}-C_{s}$, and their coupling to the line through the mutual inductance, $M$ [60] (the circuit model of SRR-loaded lines was first published in [59], and later revised in [60]). The circuit model can be transformed to the one depicted in Figure 15(b), with the indicated transformation equations. Indeed, the model of the SRR-loaded CPW is not identical to the canonical model of a CRLH transmission line depicted in Figure 5. The series branch is an inductor in series with a parallel resonator. However, the CRLH behavior of the line is accounted for by this model, as can be derived from the dispersion relation obtained from (5):

$$
\cos (\beta l)=1+\frac{L^{\prime}}{L_{p}^{\prime}}\left(1-\frac{\omega^{2}}{\omega_{p}^{2}}\right)\left(1+\frac{L_{s}^{\prime} / L^{\prime}}{1-L_{s}^{\prime} C_{s}^{\prime} \omega^{2}}\right)
$$

with $\omega_{p}$ given by

$$
\omega_{p}=\frac{1}{\sqrt{C L_{p}^{\prime} / 2}} .
$$

The main difference is the presence of a transmission zero to the left of the first (left handed) transmission band given by the resonance of the parallel resonator of the series branch. Notice that according to the transformation equations of Figure 15, this transmission zero does not exactly correspond to the resonance frequency of the SRR. The characteristic impedance is similar to that depicted in Figures 6(b) or 7(b) (depending on whether the structure is unbalanced or balanced).

(2) CSRR-Based Metamaterial Transmission Lines. Resonant-type CRLH transmission lines in microstrip technology can be implemented by etching CSRRs in the ground plane, beneath the conductor strip, and series gaps in the conductor strip, as illustrated in Figure 16 [48, 61, 62]. The circuit model of these lines is depicted in Figure 17(a), where $L$ and $C_{L}$ model the line inductance and capacitance, respectively, and the gap is modeled by a $\pi$-circuit consisting of a series capacitance, $C_{s}$, and two fringing capacitances, $C_{f}$. Finally, the CSRR is modeled by the resonant tank, $L_{c}-C_{c}$ [63] 

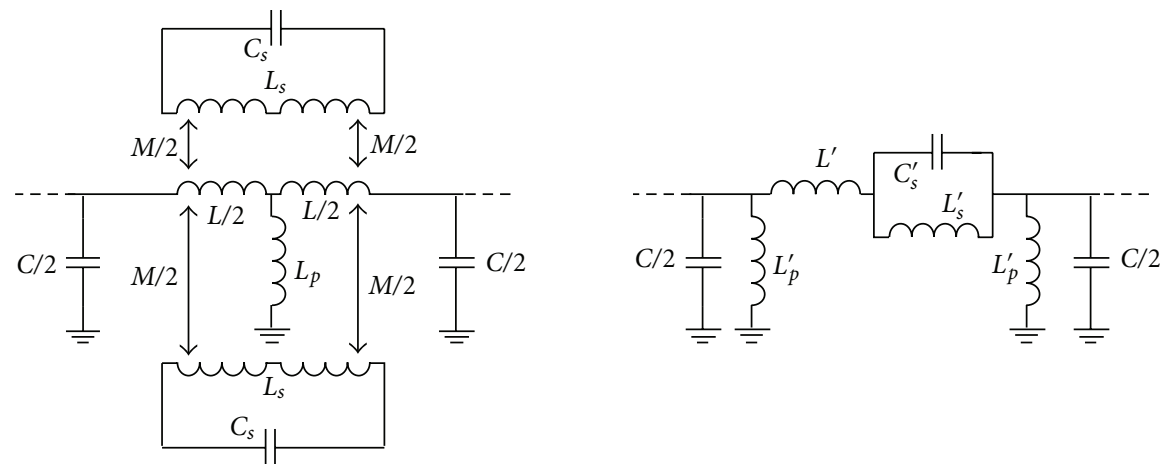

$$
L_{s}^{\prime}=2 M^{2} C_{s} \omega_{0}^{2} \frac{\left(1+L / 4 L_{p}\right)^{2}}{1+M^{2} / 2 L_{p} L_{s}} \quad C_{s}^{\prime}=\frac{L_{s}}{2 M^{2} \omega_{0}^{2}}\left(\frac{1+M^{2} / 2 L_{p} L_{s}}{1+L / 4 L_{p}}\right)^{2}
$$

(a)

$$
L^{\prime}=\left(2+L / 2 L_{p}\right) \frac{L}{2}-L_{s}^{\prime} \quad L_{p}^{\prime}=2 L_{p}+\frac{L}{2}
$$

FIgURE 15: Circuit model for the basic cell of the CRLH SRR-based CPW structure (a). Transformation of the model to a $\pi$-circuit (b). The transformation equations from (a) to (b) are indicated.

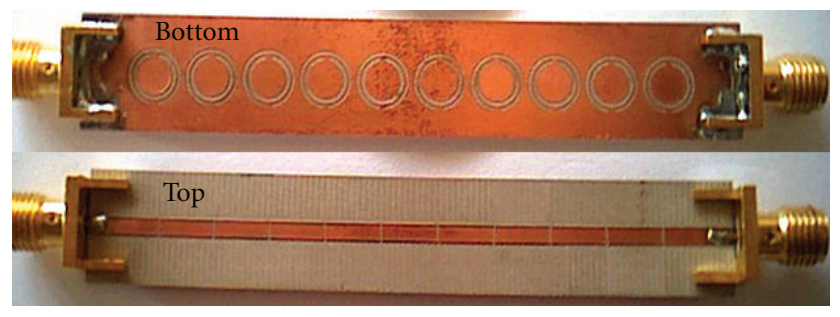

FIGURE 16: Photograph of a CRLH CSRR-based microstrip transmission line.

(The circuit model of CSRR-loaded lines was first published in [61], and then revised in [63]). This circuit model can be transformed to the model depicted in Figure 17(b), where the indicated equations have been applied. The circuit model of Figure 17(b) explains also the CRLH behavior of CSRRloaded microstrip lines. In this case, the transmission zero present to the left of the first band is given by

$$
f_{z}=\frac{1}{2 \pi \sqrt{L_{c}\left(C+C_{c}\right)}} .
$$

The dispersion relation and the characteristic impedance are given by

$$
\begin{gathered}
\cos \beta l=1-\frac{\left(C L \omega^{2} / 2\right)\left(1-\omega_{s}^{2} / \omega^{2}\right)\left(1-\omega_{p}^{2} / \omega^{2}\right)}{\left(1-\omega_{p}^{2} / \omega^{2}+C L_{c} \omega_{p}^{2}\right)}, \\
Z_{B}=\sqrt{\frac{L}{C_{c}} \frac{\left(1-\omega_{s}^{2} / \omega^{2}\right)}{\left(1-\omega_{p}^{2} / \omega^{2}\right)}-\frac{L^{2} \omega^{2}}{4}\left(1-\frac{\omega_{s}^{2}}{\omega^{2}}\right)^{2}+\frac{L}{C}\left(1-\frac{\omega_{s}^{2}}{\omega^{2}}\right)},
\end{gathered}
$$

where $\omega_{s}$ and $\omega_{p}$ are the series and shunt resonances of the circuit of Figure 17(b). For the balanced case, $\omega_{s}=\omega_{p}=\omega_{o}$, and the previous expression simplifies to

$$
Z_{B}=\sqrt{\frac{L}{C_{c}}-\frac{L^{2} \omega^{2}}{4}\left(1-\frac{\omega_{o}^{2}}{\omega^{2}}\right)^{2}+\frac{L}{C}\left(1-\frac{\omega_{o}^{2}}{\omega^{2}}\right)} .
$$

Expression (33) is very similar to (20). The difference is the presence of the last term in the square root of (33). As for the balanced transmission line based on the canonical CRLH circuit model of Figure 5, the characteristic impedance of balanced CSRR-based lines is null at the extremes of the allowed band, and it varies continuously in between. However, in resonant-type balanced lines loaded with CSRRs, the impedance is maximum above the transition frequency, $f_{o}$. Nevertheless, the impedance variation in the vicinity of the transition frequency is smooth and this is interesting to preserve matching in a wide band. The variation of $Z_{p}, Z_{s}$, and $Z_{B}$ with frequency for the transmission line model of Figure 17(b) in the balanced case is depicted in Figure 18. Figure 19 shows a designed balanced CRLH CSRR-based microstrip transmission line unit cell [15]. The interdigital capacitor has been necessary to achieve the required capacitance for balancing the structure. The dispersion relation and the frequency response are also depicted in the figure, where it can be appreciated that the balance condition is roughly achieved. At high frequencies, the model of Figure 17 is no longer valid, and the predictions of this model deviate from electromagnetic simulation and measurement.

(3) OSRR- and OCSRR-Based Metamaterial Transmission Lines. Resonant type CRLH transmission lines can also be implemented by combining open SRRs (OSRRs [49]) and open CSRRs (OCSRRs [51]). The typical topology and circuit model of a OSRR and an OCSRR loading a CPW structure are depicted in Figures 20 and 21 [16]. Essentially, the OSRR and the OCSRR are described by a series and parallel resonator, respectively. However, the host line introduces some phase shift that must be taken into account for an accurate modelling of the structures. According to Figures 20 and 21, the models of the structures are composed of the resonators, plus a parasitic capacitance and a parasitic inductance for the OSRR-loaded CPW and for the OCSRR-loaded CPW, respectively. Due to the presence 


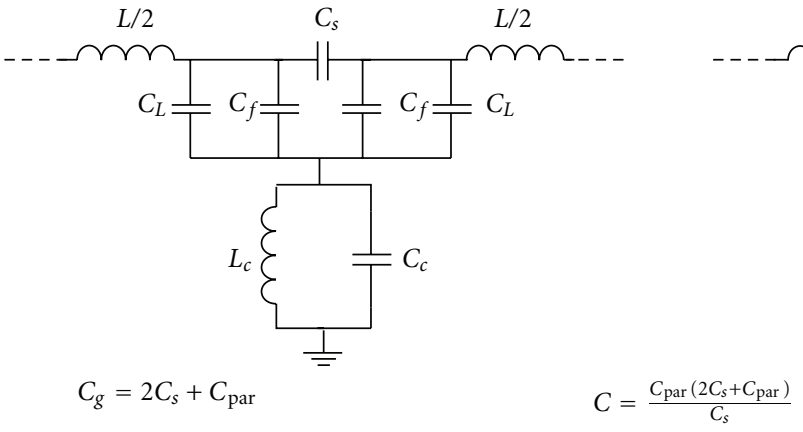

(a)

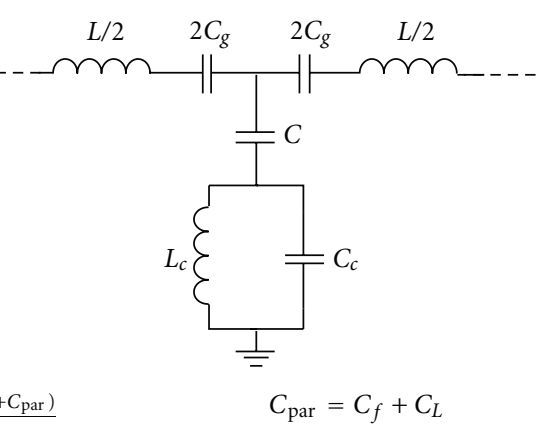

(b)

FIgURE 17: Circuit model for the basic cell of the CRLH CSRR-based microstrip structure (a). Transformation of the model to a T-circuit (b). The transformation equations from (a) to (b) are indicated.

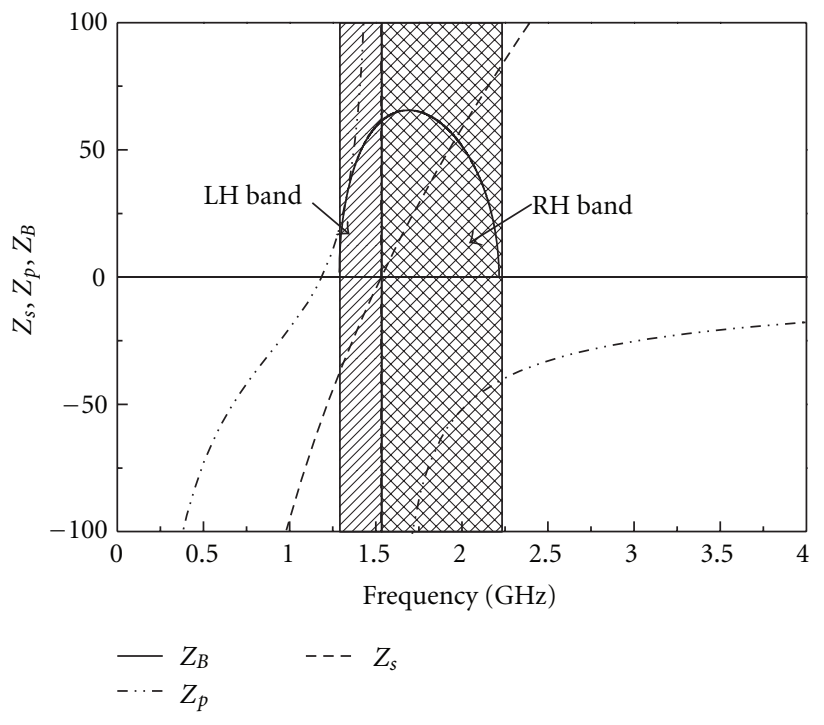

FIGURE 18: Representation of the series, $Z_{s}$, shunt, $Z_{p}$, and characteristic impedance (real part), $Z_{B}$, for a CRLH transmission line corresponding to the model of a balanced CSRR-based structure. Electrical parameters are $L=45 \mathrm{nH}, C_{g}=0.48 \mathrm{pF}, C=10 \mathrm{pF}$, $C_{c}=3 \mathrm{pF}$, and $L_{c}=3 \mathrm{nH}$. The depicted values of $Z_{s}$ and $Z_{p}$ are actually the reactances. The transition frequency has been set to $f_{o}=1.5 \mathrm{GHz}$. From [15]; copyright (C2007 IEEE, reprinted with permission.

of these parasitics, the implementation of canonical CRLH structures (modelled by the circuit of Figure 5), by cascading OSRR and OCSRR-loaded CPW sections, is not possible. However, these parasitics are small and, in practice, the OSRR- and OCSRR-based lines exhibit a canonical CRLH behaviour to a good approximation. The main difference as compared to SRR- or CSRR-loaded lines is the absence of the transmission zero to the left of the left-handed band. Another important aspect concerns the accuracy of the circuit models. Due to the smaller electrical size of the open particles (OSRRs and OCSRRs), the circuit models of the OSRR- and OCSRR-based structures provide an accurate description of them up to higher frequencies, as compared to the models of SRR- or CSRR-loaded lines. Figure 22 depicts a typical OSRR and OCSRR CRLH CPW transmission lines. The frequency response, the characteristic impedance, and the dispersion diagram are also depicted in Figure 22. The structure is quasibalanced as derived from the small gap in the dispersion diagram (discrepancies with measurement are due to fabrication-related tolerances). It can be seen that the model describes the structure to a good approximation in the frequency range shown.

The implementation of higher order CRLH transmission lines has been mainly done by combining semilumped and lumped elements $[64,65]$. Nonetheless, fully planar order4 CRLH structures implemented by means of OSRRs and OCSRRs have been demonstrated [22].

The advantages of metamaterial transmission lines mainly concern the fact that thanks to the presence of the reactive elements, both the characteristic impedance and the phase constant can be engineered with further flexibility than in conventional lines. Thus, metamaterial transmission lines are useful for the design of microwave components on the basis of impedance and dispersion engineering. Miniaturization is another key aspect of these artificial lines. Finally, as long as metamaterial transmission lines exhibit pass bands and stop bands, they are also useful for microwave filter design. These advantages will be fully appreciated in the next sections, devoted to the applications.

\section{Applications of Metamaterial Transmission Lines (Part I): Broadband Filters and Multiband Components}

Metamaterial transmission lines have found numerous applications in wireless communications, including guided wave components and antennas. Among guided wave components, metamaterial transmission lines have been applied to the design of enhanced bandwidth components [66-73], multiband components $[16,22,64,65,74-80]$, tightly coupled couplers [81-85], resonators [86], filters and diplexers [16-18, 44, 49-52, 56, 87-104], and so forth. In the field of antennas, the design of leaky wave antennas with scanning capability has been a notable contribution [14, 105-112] (however, novel antennas based on metamaterial concepts 


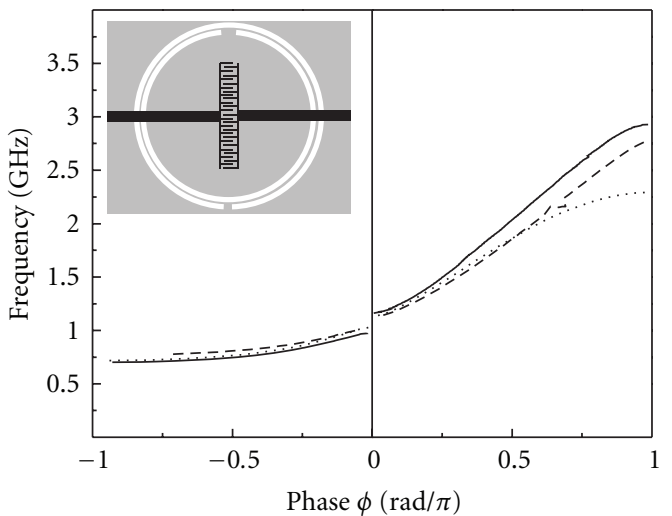

..... Electrical simulation $\quad$ Measurement - - - EM simulation

(a)

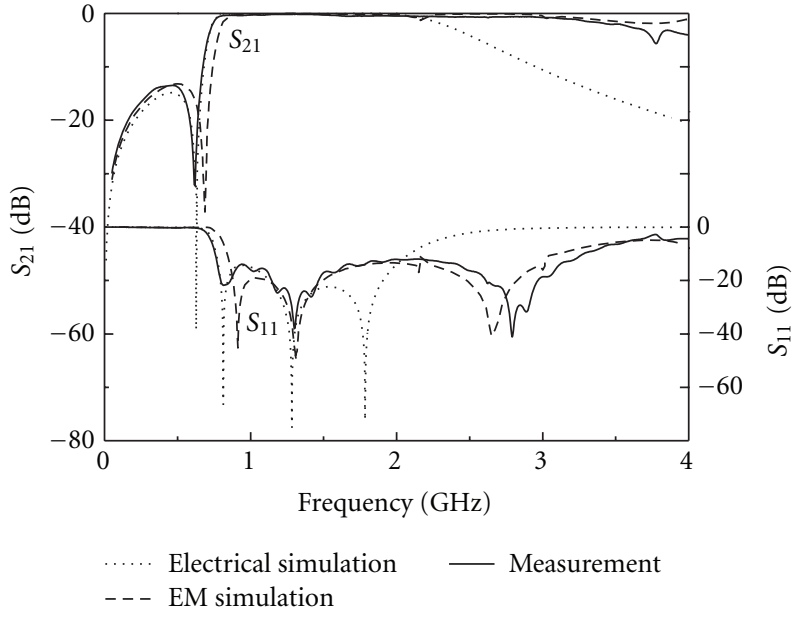

(b)

FIGURE 19: Balanced CRLH cell based on a microstrip line loaded with CSRRs (inset) and dispersion diagram (a), and frequency response (b). The structure has been implemented in the Rogers RO3010 substrate with dielectric constant $\varepsilon_{r}=10.2$ and thickness $h=1.27 \mathrm{~mm}$. Dimensions are line width $W=0.8 \mathrm{~mm}$, external radius of the outer ring $r_{\mathrm{ext}}=7.3 \mathrm{~mm}$, rings width $c=0.4 \mathrm{~mm}$, and rings separation $d=0.2 \mathrm{~mm}$; the interdigital capacitor, formed by 28 fingers separated by $0.16 \mathrm{~mm}$, has been used to achieve the required capacitance value. From [15]; copyright (C) 2007 IEEE, reprinted with permission.

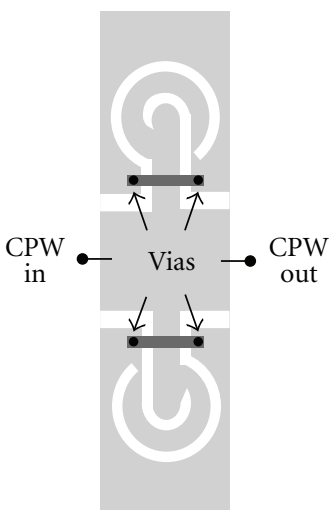

(a)

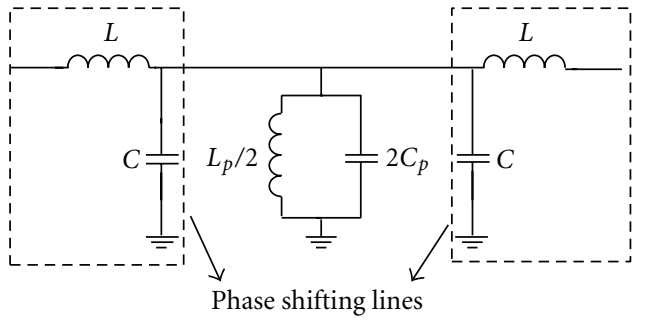

(b)

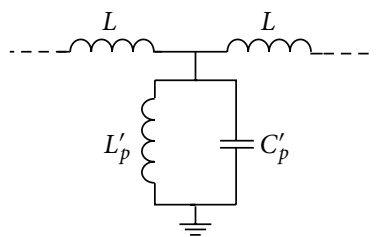

(c)

FIGURE 20: Layout (a), circuit model (b), and simplified circuit model (c) of a CPW transmission line loaded with a pair of OCSRRs, where $C_{p}^{\prime}=2 C_{p}+2 C$ and $L_{p}^{\prime}=L_{p} / 2$. The backside strips (in dark grey) connecting the different ground plane regions are necessary to prevent the slot mode of the CPW and the second resonance of the open complementary split ring resonators.

and metamaterial resonators have also been proposed in recent years [7, 113-125]). Let us consider in certain detail the application of CRLH transmission lines to the design of broadband filters and multiband components.

3.1. Broadband Filters. As long as metamaterial transmission lines exhibit pass band and stop bands, their application to the implementation of filters seems to be straightforward. The first stop band filters were simply implemented by loading a host transmission line with SRRs or CSRRs or with other related resonators [52]. For the design of band pass filters, a new approach was presented in [17], and subsequently used in [18] for the design of broadband filters. Essentially, the filters reported in $[17,18]$ are periodic structures based on the so-called hybrid approach, where a host line (microstrip line) is loaded with CSRRs, series gaps, and shunt stubs. The presence of shunt stubs provides further design flexibility and the frequency response of the filter can be tailored. For more details on the design of these filters, the author recommends papers [17] and [18]. Nevertheless, for completeness, we depict in Figure 23 a band pass filter with moderate bandwidth, compared to a conventional coupled line band pass filter with comparable performance (the filter response is also depicted in the figure). This design strategy 

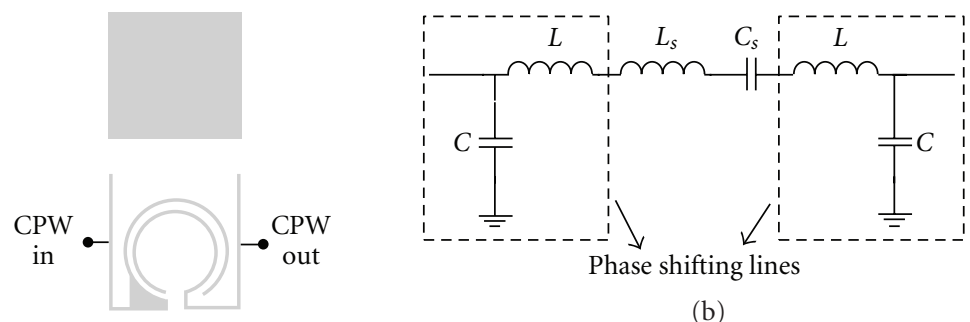

(b)

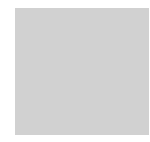

(a)

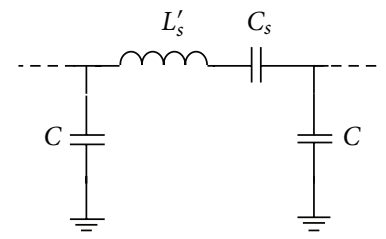

(c)

FiguRe 21: Layout (a), circuit model (b), and simplified circuit model (c) of a CPW transmission line loaded with a series connected OSRR $\left(L_{s}^{\prime}=L_{s}+2 L\right)$.

combines good filter performance with small size, but filter synthesis is complex.

Balanced CSRR-based CRLH lines for the realization of broad band filters have also been proposed [18]. In such filters, selectivity at the lower edge of the pass band is good, but the upper band is scarcely controllable, as it can be appreciated in Figure 24.

Indeed, the circuit model of the unit cell of a canonical CRLH transmission line (Figure 5) is also the canonical circuit of an order- 3 band pass filter. Based on this idea, we have implemented broad band filters by combining OSRRs and OCSRRs. The OSRRs account for the series resonators, whereas the OCSRRs account for the shuntconnected parallel resonators. These open resonators are wideband resonators by nature and therefore are specially suited to obtain broadband responses. Several papers report the design of broadband filters based on the combination of these open resonators $[16,102,103]$. To illustrate the potential of the approach, we report here the design of a Chebychev band pass filter with central frequency at $2 \mathrm{GHz}$, $50 \%$ fractional bandwidth and $0.05 \mathrm{~dB}$ ripple (Figure 25) [19]. Good agreement between the circuit simulation, the electromagnetic simulation, and the measured frequency response is achieved in the region of interest. Dimensions are $0.24 \lambda \times 0.17 \lambda$. Measured in-band insertion losses $(-1.09 \mathrm{~dB}$ at the transmission band center) are mainly due to conductor losses and are caused by the critical dimensions of the structure (conductor and dielectric losses have been excluded in the electromagnetic simulation to ensure a proper design). More details on the design of this type of filters can be found in $[16]$.

3.2. Multiband Components. Conventional lines are singleband structures by nature. That is, they exhibit the required phase at a single frequency. Typically, distributed circuits make use of transmission lines and stubs of certain electrical length and, therefore, these circuits do also exhibit their functionality at a single frequency. However, as long as metamaterial transmission lines exhibit a controllable dispersion diagram and characteristic impedance, the previous limitation can be circumvented, and dual-band and multiband microwave devices can be implemented. Specifically, by adding reactive elements to the line, it is possible to force the required characteristic impedance and phase at more than one frequency. To illustrate the potentiality of CRLH transmission lines to the design of multiband components, let us consider the unit cell of Figure 5 and force a certain characteristic impedance and electrical length at two frequencies (dual band functionality). In many applications, the sign of the electrical length is not an issue. Therefore, we can use the first (left handed) band for the lower frequency and the second (right handed) band for the upper frequency. Moreover, many microwave circuits are based on $90^{\circ}$ transmission lines; thus, we will consider the synthesis of transmission lines exhibiting a characteristic impedance and phase (electrical length) of $Z_{A},-90^{\circ}$ at $f_{1}$, and $Z_{A},+90^{\circ}$ at $f_{2}$. Using expressions (5) and (6), the series and shunt reactances of the T-circuit of Figure 5 are found to be

$$
\begin{gathered}
\chi_{s}\left(\omega_{1}\right)=-Z_{A}, \\
\chi_{p}\left(\omega_{1}\right)=Z_{A}, \\
\chi_{s}\left(\omega_{2}\right)=Z_{A}, \\
\chi_{p}\left(\omega_{2}\right)=-Z_{A}
\end{gathered}
$$

with $Z_{s}(\omega)=j \chi_{s}(\omega)$ and $Z_{p}(\omega)=j \chi_{p}(\omega)$. From (34a)-(34d), the elements of the circuit model can be easily inferred. Indeed, expressions (34a)-(34d) can be generalized for a multiband structure as follows:

$$
\begin{gathered}
\chi_{s}\left(\omega_{n}\right)=(-1)^{n} Z_{A}, \\
\chi_{p}\left(\omega_{n}\right)=(-1)^{n+1} Z_{A},
\end{gathered}
$$




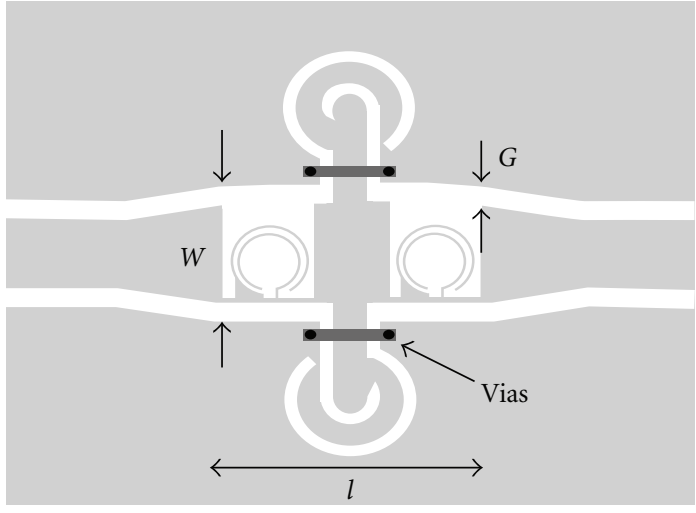

(a)

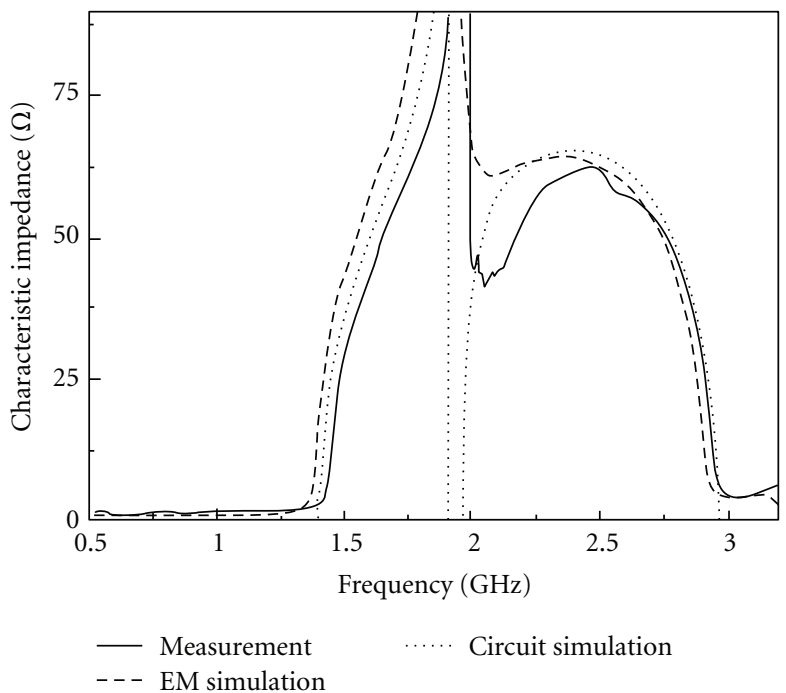

(c)

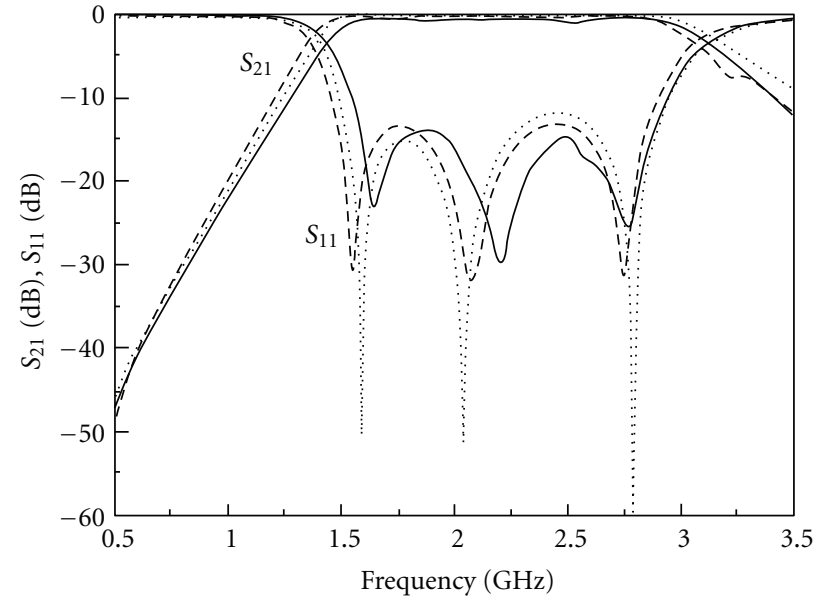

(b)

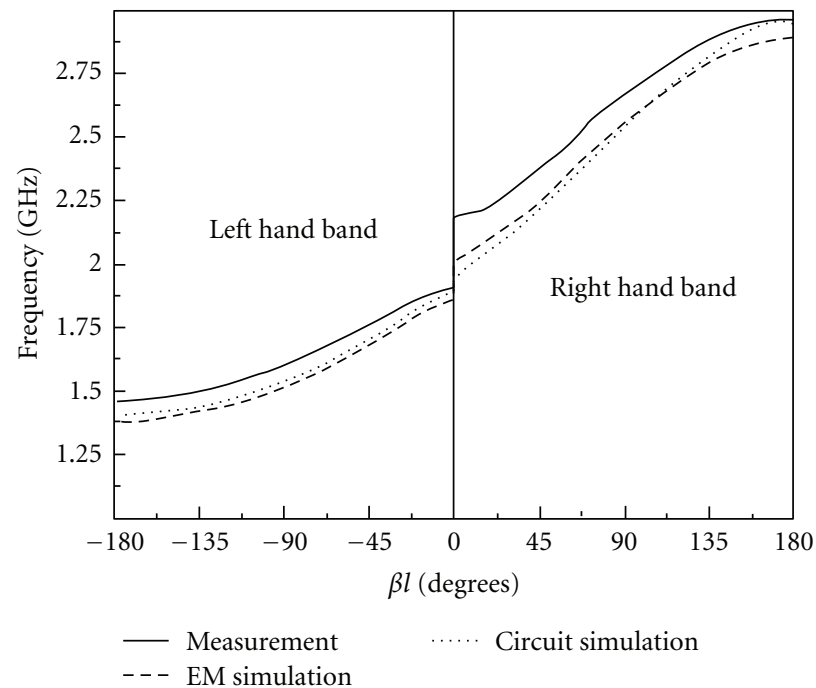

(d)

FIGURE 22: (a) Topology of the unit cell of a CPW CRLH transmission line based on a combination of series connected OSRRs in the external stages and a pair of shunt-connected OCSRRs in the central stage; (b) frequency response; (c) characteristic impedance; (d) dispersion diagram. The considered substrate is the Rogers RO3010 with thickness $h=1.27 \mathrm{~mm}$ and dielectric constant $\varepsilon_{r}=10.2$. Dimensions are $l=12.3 \mathrm{~mm}, W=5 \mathrm{~mm}$, and $G=1.28 \mathrm{~mm}$. For the open complementary split ring resonator: $r_{\mathrm{ext}}=2.9 \mathrm{~mm}, c=0.5 \mathrm{~mm}$, and $d=1.2 \mathrm{~mm}$. For the open split ring resonators: $r_{\mathrm{ext}}=2 \mathrm{~mm}, c=d=0.2 \mathrm{~mm}$. From [16]; copyright (C) 2009 IEEE, reprinted with permission.

where $n=1,2,3,4 \ldots$ is the frequency index. Obviously, (35a)-(35b) can be solved if the number of elements of the series and shunt branch coincides with the frequency index.

To illustrate the potential of CRLH transmission lines to the design of multiband components, let us consider the design of a dual band Y-junction power divider based on a structure similar to that depicted in Figure 22, that is, based on OSRRs and OCSRRs. The power divider is based on a $35.35 \Omega$ impedance inverter functional at $f_{1}=2.4 \mathrm{GHz}$ and $f_{2}=3.75 \mathrm{GHz}$. Hence, the artificial line has been designed with an electrical length of $-90^{\circ}$ at $f_{1}$ and $+90^{\circ}$ at $f_{2}$. The details of the inverter design, using expressions (34a)-(34d) and the models reported in Figures 20 and 21, are given in [16]. Figure 26 depicts the designed inverter, its dispersion diagram, and its characteristic impedance (the target characteristic impedance and phase at the design frequencies have been achieved). To implement the divider, two output access lines have been added, as shown in Figure 27. The characterization of this device is also depicted in Figure 27, where the functionality at the operating frequencies is achieved.

Other dual-band components based on CSRR-loaded lines and CL-loaded lines (lines loaded with series capacitances and shunt inductances) have been reported in [74-80]. We would like also to mention that quad-band components (power dividers and branch line couplers) based on a fully planar approach, by using open resonators and other planar resonators, have been demonstrated [22]. A quad-band power divider operating at the GSM $\left(f_{1}=\right.$ $\left.0.9 \mathrm{GHz}, f_{4}=1.8 \mathrm{GHz}\right)$ and the GPS $\left(f_{2}=1.17642 \mathrm{GHz}\right.$, 


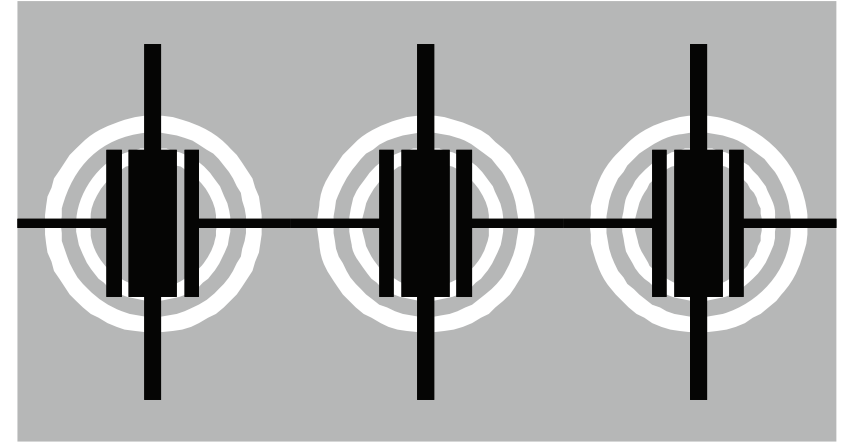

(a)

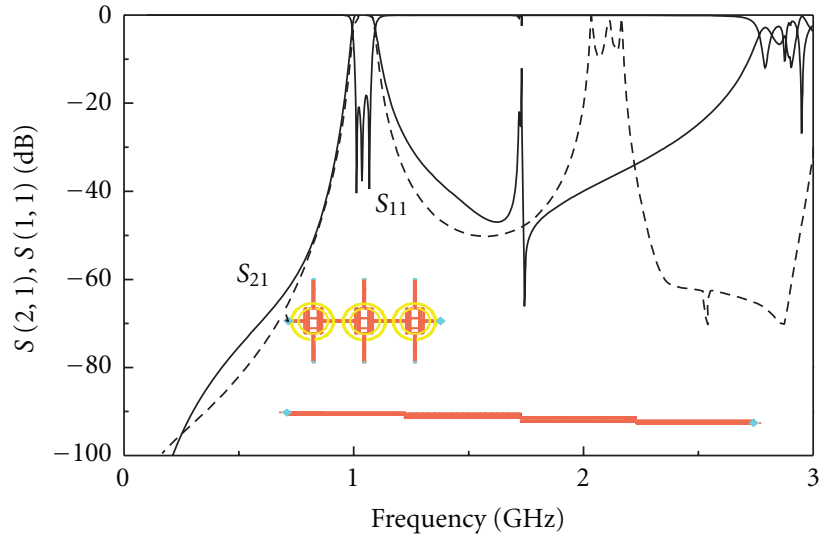

(b)

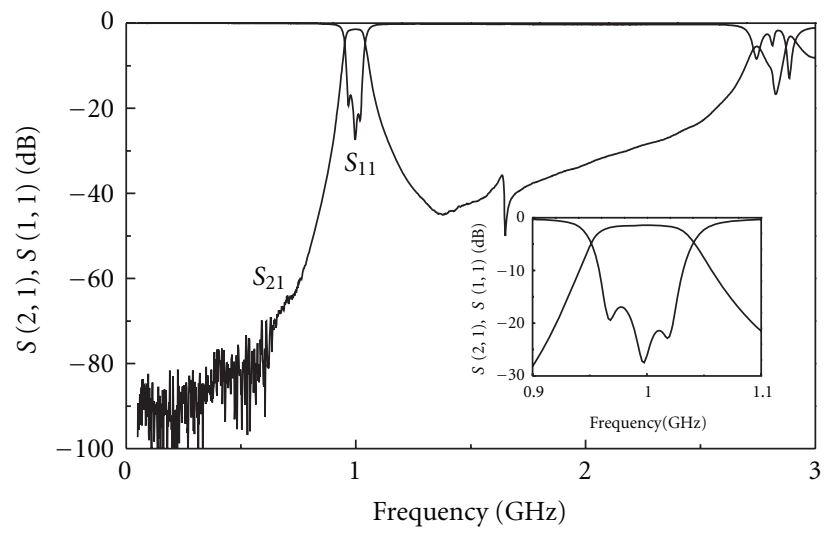

(c)

FIGURE 23: Layout of a designed and fabricated band pass filter based on the hybrid approach (a), simulated (b), and measured (c) insertion and return losses. Total device length excluding access lines is $4.56 \mathrm{~cm}$. In (b), the simulated insertion loss for a conventional order-3 coupled line filter with similar performance is depicted (dashed line). From [17]; copyright ( 2006 IEEE; reprinted with permission.

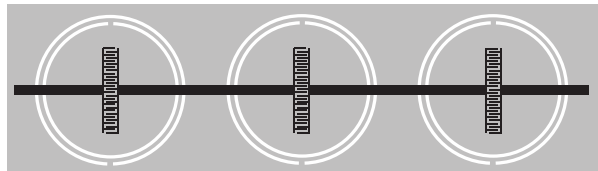

(a)

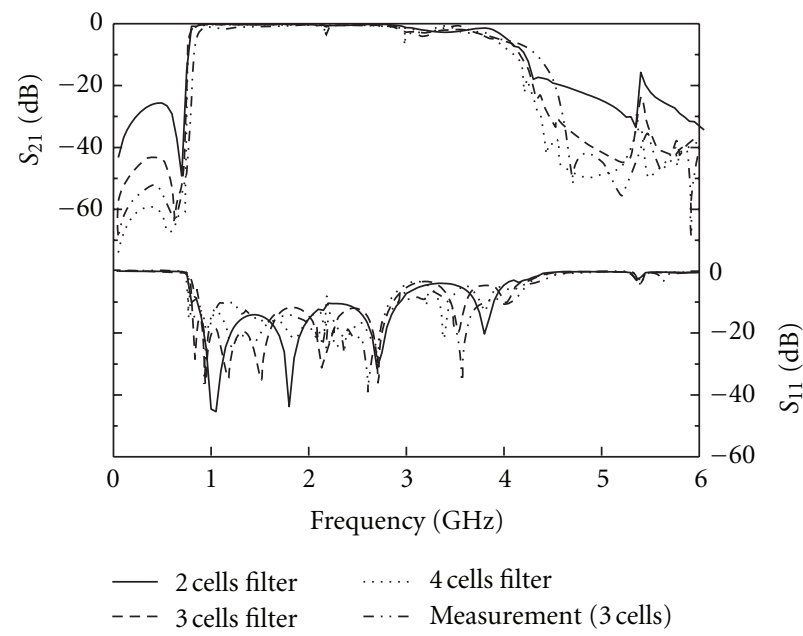

(b)

FIGURE 24: Layout of a microstrip filter formed by three balanced CRLH CSRR-based cells (a) and measured frequency response (b). In (b) are also depicted the simulated insertion and return loss for a two-, three-, and four-stage device filters. The metallic parts are depicted in black in the top layer, and in grey in the bottom layer. Dimensions are total length $l=55 \mathrm{~mm}$, line width $W=0.8 \mathrm{~mm}$, external radius of the outer rings $r=7.3 \mathrm{~mm}$, rings width $c=0.4 \mathrm{~mm}$, and rings separation $d=0.2 \mathrm{~mm}$; the interdigital capacitors are formed by 28 fingers separated $0.16 \mathrm{~mm}$. From [18]; copyright (C) 2007 IEEE; reprinted with permission. 

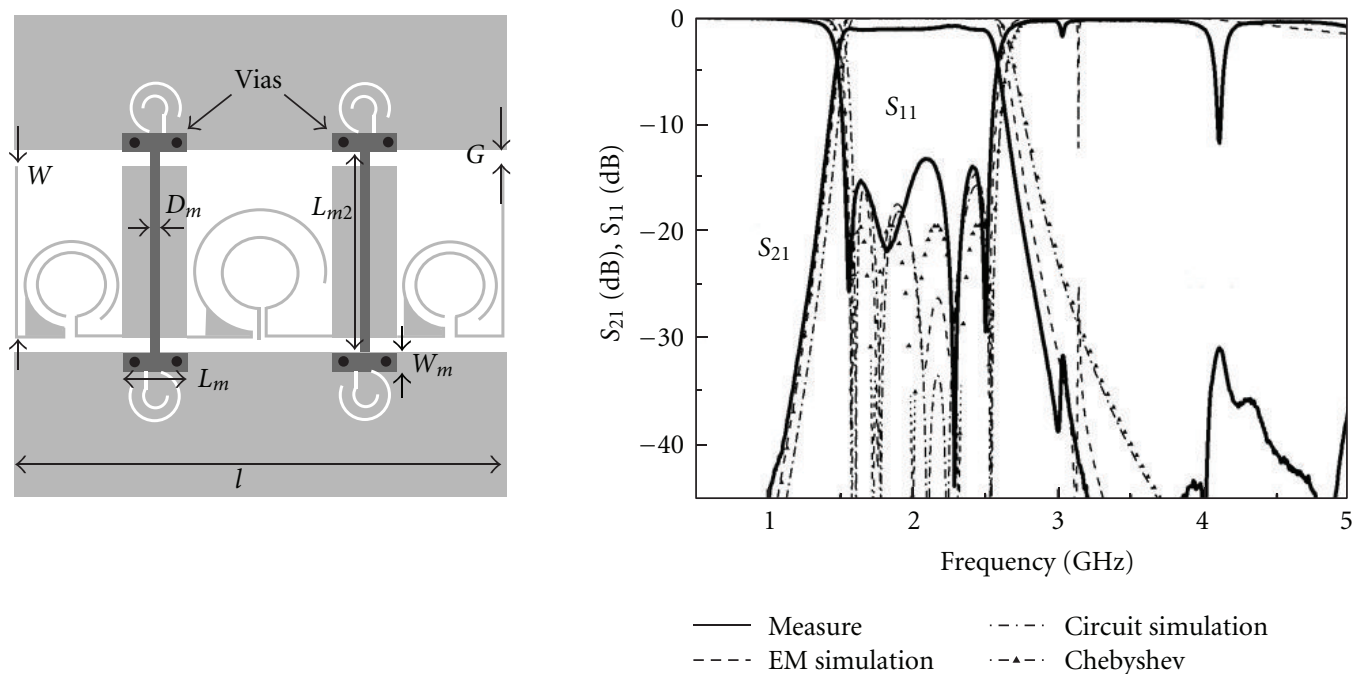

(a)

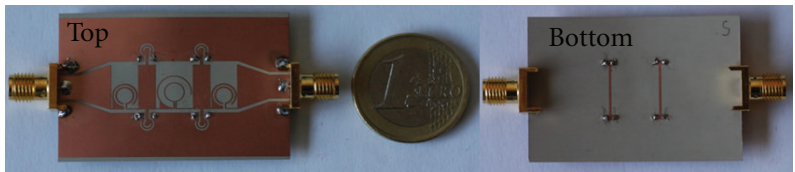

(b)

FIGURE 25: Topology (a), photograph (b), and frequency response (c) for the designed fifth-order filter implemented by means of OSRRs and OCSRRs. The substrate is the Rogers RO3010 with thickness $h=0.254 \mathrm{~mm}$ and measured dielectric constant $\varepsilon_{r}=11.2$. Dimensions are $l=25 \mathrm{~mm}, W=9.23 \mathrm{~mm}$, and $G=0.71 \mathrm{~mm}$. For the external OSRRs: $r_{\text {ext }}=2.5 \mathrm{~mm}, c=0.3 \mathrm{~mm}$, and $d=0.35 \mathrm{~mm}$. For the central OSRR: $r_{\mathrm{ext}}=3.4 \mathrm{~mm}, c=0.16 \mathrm{~mm}, d=1.24 \mathrm{~mm}, L_{m}=3.2 \mathrm{~mm}, W_{m}=0.96 \mathrm{~mm}, D_{m}=0.4 \mathrm{~mm}$, and $L_{m 2}=10.64 \mathrm{~mm}$. For the OCSRRs: $r_{\text {ext }}=1.4 \mathrm{~mm}, c=d=0.3 \mathrm{~mm}$. The element values that have been used in reference of Figures 20 and 21 are for the external OSRRs: $C=0.207 \mathrm{pF}, C_{s}=0.763 \mathrm{pF}$, and $L_{s}^{\prime}=8.501 \mathrm{nH}$; for the central OSRR: $C=0.274 \mathrm{pF}, C_{s}=0.436 \mathrm{pF}$, and $L_{s}^{\prime}=13.118 \mathrm{nH}$; for the OCSRRs: $L=0.474 \mathrm{nH}, C_{p}^{\prime}=4.5 \mathrm{pF}$, and $L_{p}^{\prime}=1.224 \mathrm{nH}$. From [19]; copyright @ 2011 IEEE; reprinted with permission.

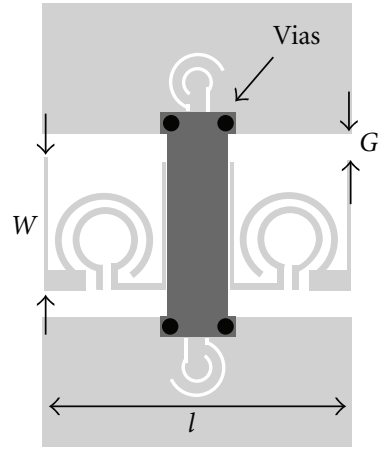

(a)

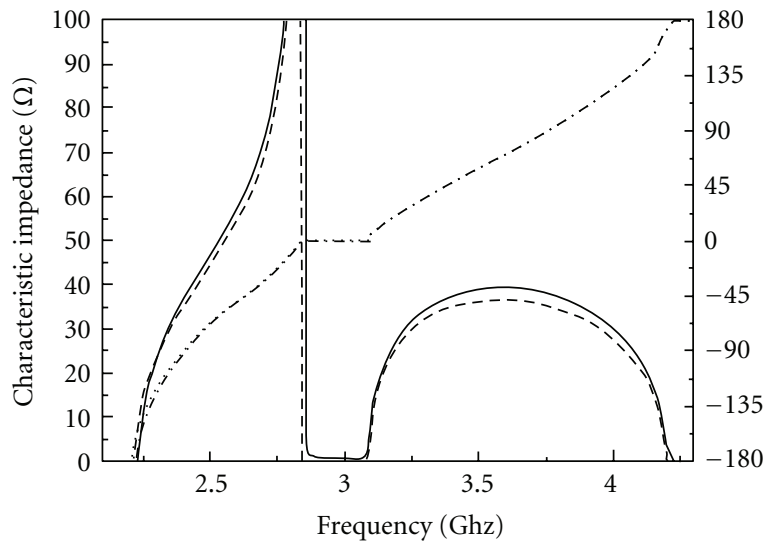

..... $\beta l$ circuit simulation $\quad---$ Zo circuit simulation
$\ldots$-.. $\beta l$ EM simulation

(b)

FIGURE 26: (a) Layout of the dual-band impedance inverter based on a combination of series connected OSRRs in the external stages and a pair of shunt-connected OCSRRs in the central stage; (b) circuit and electromagnetic simulation. The substrate is the Rogers RO3010 with thickness $h=0.635 \mathrm{~mm}$ and dielectric constant $\varepsilon_{r}=10.2$. Dimensions are $l=9 \mathrm{~mm}, W=4 \mathrm{~mm}, G=0.74 \mathrm{~mm}$. For the open complementary split ring resonator, $r_{\mathrm{ext}}=0.9 \mathrm{~mm}, c=0.2 \mathrm{~mm}$, and $d=0.2 \mathrm{~mm}$. For the open split ring resonators, $r_{\mathrm{ext}}=1.5 \mathrm{~mm}, c=0.3 \mathrm{~mm}$, and $d=0.2 \mathrm{~mm}$. The wide metallic strip in the back substrate side has been added in order to enhance the shunt capacitance of the open complementary split ring resonator stage, as required to achieve the electrical characteristics of the device. From [16]; copyright (C) 2009 IEEE, reprinted with permission. 


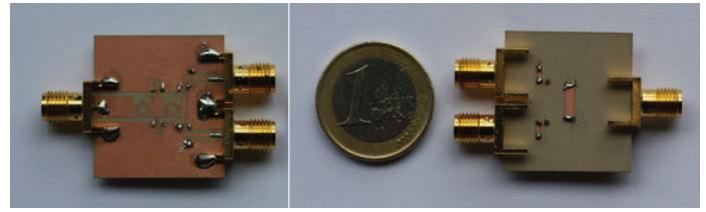

(a)

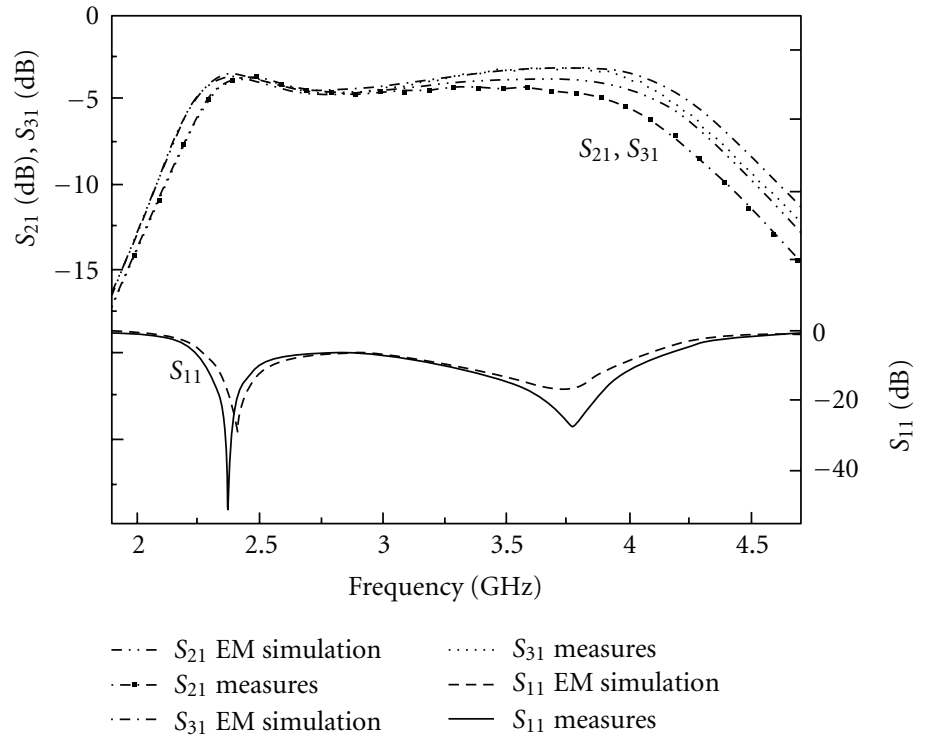

(b)

FIGURE 27: Photograph of the fabricated dual-band power splitter (a) and frequency response (b). From [16]; copyright (c) 2009 IEEE, reprinted with permission.

$f_{3}=1.57542 \mathrm{GHz}$ ) frequency bands is depicted in Figure 28 as an illustrative example. Finally, SIW technology seems to be a good candidate for the implementation of quad-band components [126].

\section{Applications of Metamaterial Transmission Lines (Part II): UHF-RFID Tags}

Radiofrequency identification (RFID) is a method for identification and gathering information of objects, consisting of a reader (interrogator) and a tag (transponder) [127131]. Ultrahigh frequency (UHF) passive tags, which are attached to the identifying objects, contain the antenna and an integrated circuit (where the information related to the object is stored), both supported by a plastic substrate (inlay). The UHF-RFID band covers the frequency span from $840 \mathrm{MHz}$ up to $960 \mathrm{MHz}$, but the regulated operating frequencies worldwide are different. Thus, for instance, Europe operates at $f_{1}=867 \mathrm{MHz}$, whereas in USA the regulated frequency is $f_{2}=915 \mathrm{MHz}$. In UHF-RFID tags, conjugate matching between the antenna and the chip is important in order to obtain a good figure of merit of the $\mathrm{tag}$, that is, the read range, which is given by [132]

$$
r=\frac{\lambda}{4 \pi} \sqrt{\frac{\mathrm{EIRPG}_{r} \tau}{P_{\text {chip }}}}
$$

where $\lambda$ is the wavelength, $G_{r}$ is the gain of the receiving tag antenna, $\tau$ is the power transmission coefficient, $P_{\text {chip }}$ is the threshold activation power of the chip, and EIRP is the equivalent isotropic-radiated power, which is determined by local country regulations. Conjugate matching has direct influence on the power transmission coefficient, and therefore in the read range. Typically, the input impedance of the chip is modelled by a parallel combination of a resistance and a capacitance. Therefore, conjugate matching means to cancel the reactive part of the chip impedance with the impedance seen from the chip port (i.e., that corresponding to the antenna cascaded to the impedance matching network, if it is present), see Figure 29. Obviously, conjugate matching cannot be achieved in the whole UHFRFID band. However, by means of a matching network, it is possible to achieve conjugate matching in more than one band. Specifically, it has been demonstrated that conjugate matching at the European and USA bands can be achieved by using a metamaterial-based structure, that is, a transmission line loaded with an SRR [133]. Once the impedance of the antenna and the chip impedance at the operating frequencies are known, the requirements for the artificial transmission line (impedance matching network) are also known. Let us consider that the impedance of the antenna at the design frequencies is $Z_{a}\left(f_{1}\right)=1.65-j 67.7 \Omega$ and $Z_{a}\left(f_{2}\right)=1.13-j 59.6 \Omega$, and that the considered chip exhibits an input impedance of $Z_{I C}\left(f_{1}\right)=22-j 404 \Omega$ and $Z_{I C}\left(f_{2}\right)=16-j 380 \Omega$, at the design frequencies. With these values, the requirements of the artificial transmission line are characteristic impedance $Z_{B 1}=91.18 \Omega$ and electrical length $\phi_{1}=\beta l=113.9^{\circ}$ at $f_{1}$, and $Z_{B 2}=84.67 \Omega$ and $\phi_{2}=$ $\beta l=112.6^{\circ}$ at $f_{2}$. These values can be achieved by means of a transmission line loaded with an SRR. The procedure to design such artificial line consists of designing a microstrip line with the mean value of impedance and phase shift at the central frequency. The introduction of the SRR causes a perturbation in both the characteristic impedance and the phase shift in the vicinity of their resonance frequency (tuned at the central frequency between $f_{1}$ and $f_{2}$ ), and as long as the two operating frequencies are close, and the phase and characteristic impedance requirements at these frequencies 

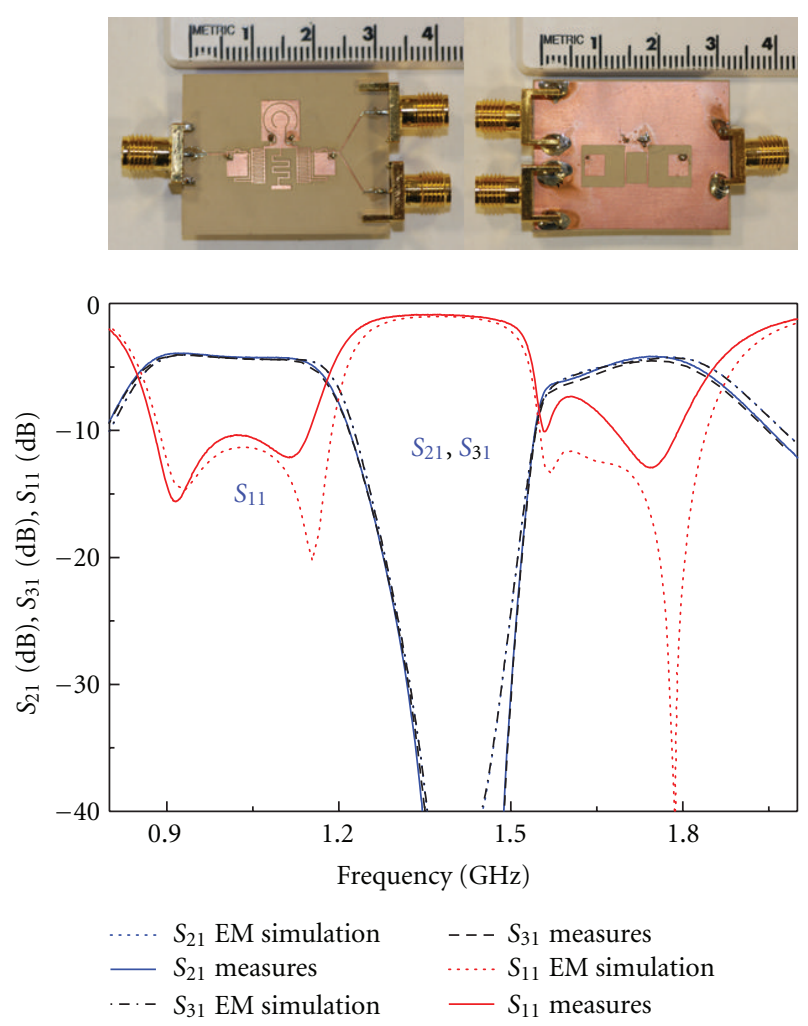

FIGURE 28: Photograph of the fabricated quad-band power splitter and simulated and measured frequency response. From [22]; copyright (C2010 IEEE, reprinted with permission.

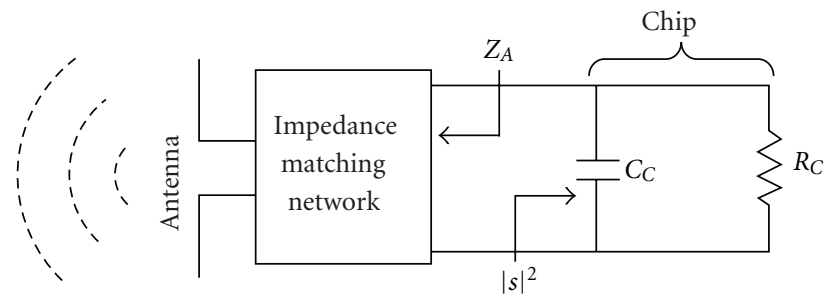

FIgURE 29: Schematic of a typical UHF-RFID tag in the most general case. An impedance matching network is located in between the chip (modeled as a $R C$ parallel load) and the tag antenna. $|s|^{2}$ is the power reflection coefficient, which is related to the power transmission coefficient according to $\tau=\left(1-|s|^{2}\right)$.

are similar, it is possible to achieve the required values by introducing the SRR. The detailed procedure, based on a perturbation method, is reported in [133]. Figure 30 depicts the layout of the fabricated antenna and matching network. The antenna is a slot antenna with the input impedance at $f_{1}$ and $f_{2}$ given above. In this implementation, the chip has not been included; hence, it has been modeled with a port with frequency-dependent impedance. This structure has been simulated through the Agilent Momentum commercial software and the prototype has been measured by means of the Agilent E8364B vector network analyzer. Since the impedance of the measuring probes is $50 \Omega$ (i.e., do not correspond to the chip impedance), a renormalization of the port impedance has been necessary. This procedure has been carried out by means of a deembedding process and resimulation with the chip impedance by means of the commercial software Agilent ADS. Specifically, we obtained the measured return loss, $S_{11}$, seen from the input of the impedance matching network (see Figure 30(a)), obviously with a $50 \Omega$ reference impedance. These results were then exported to the Agilent ADS, from which it was possible to infer the power wave reflection coefficient, $s,[134,135]$ by considering the impedance of the integrated circuit as reference impedance of the input port. Figure 30(b) illustrates the simulated (electromagnetic simulation with losses) and measured (through the explained procedure) reflection coefficient for the fabricated structure, where it can be appreciated that functionality at the design frequencies is achieved.

The procedure to achieve conjugate matching at two frequencies is efficient, but it requires a matching network. Alternatively, we can directly couple the resonator to the antenna and thus modify the antenna impedance in order to achieve nearly conjugate matching at the required frequencies [23]. This strategy has been carried out by considering meander line antennas (MLAs) and folded dipoles. Figure 31 shows the photograph of a designed dual-band tag (including the chip) based on a MLA loaded with a spiral resonator. The read range has been measured through the experimental setup available in our laboratory, according to the procedure reported in [23]. It can be appreciated that the read range at the European and USA frequencies is above $6 \mathrm{~m}$ for the dual-band tag, whereas for the tag without the coupled resonator, the read range at the operating frequency is severely degraded (this conventional single-band tag has been designed to exhibit maximum read range at the central frequency between the European and USA frequencies).

Other dual-band tags are present in the literature, but the reported implementations have been mainly oriented to be operative at different spectral bands (for instance HF and UHF, or UHF and microwave) [136-138], rather than being considered for operating at different frequencies within a certain band $[23,139-141]$. In the latter case, there are few experimental works available in the literature $[23,139]$. Even though the dual-band approach is potentially viable, the detuning problem (frequency variations caused by the attached objects) is still present in dual-band tags, and hence broadband (global band) tags seem to be the more convenient solution if detuning effects appear. There are available global band commercial tags, but there are intrinsic limitations for bandwidth enhancement (this aspect is discussed in [142]).

To end this section, we would like to mention that chipless RFID tags do also exist [143-149] and have lower cost. Two strategies are mainly used: (i) time-domain reflectometry, where data is stored in defects placed in a delay line, or (ii) RF bar codes, where data is stored in a transmission line by coupling to it resonators tuned at different frequencies. Metamaterial concepts have also been used for the implementation of this type of tags $[148,149]$. 


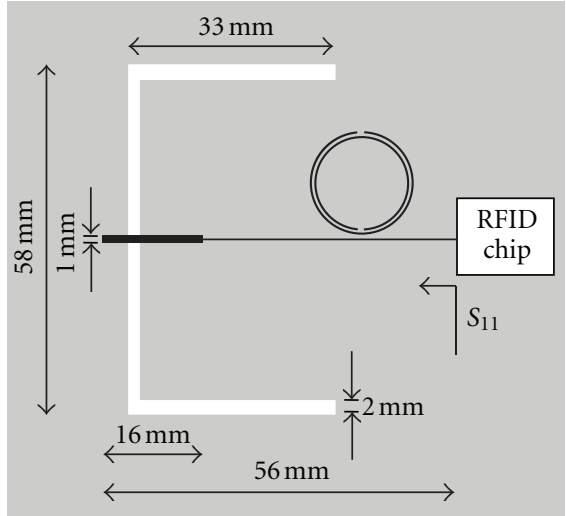

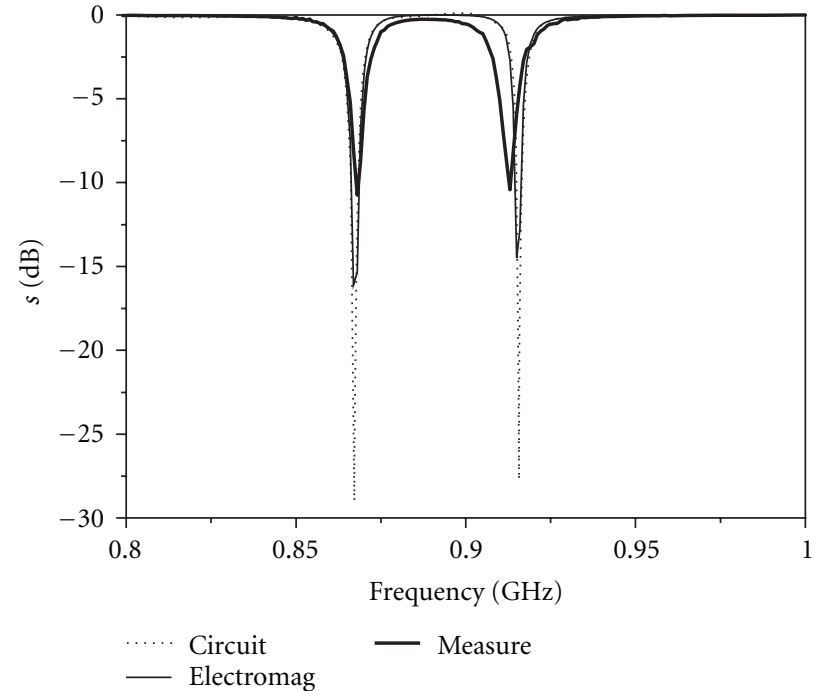

(b)

FIGURE 30: (a) Topology of the designed system consisting of the dual-band matching network between the slot antenna and RFID chip. The metallic parts are depicted in black on the top layer and in gray in the bottom layer. The slot antenna is etched on the bottom layer and depicted in white. (b) Electromagnetic simulation (by including losses), and measured return loss of the fabricated device. The considered substrate is the Rogers $R O 3010$ with dielectric constant $\varepsilon_{r}=10.2$ and thickness $h=1.27 \mathrm{~mm}$.

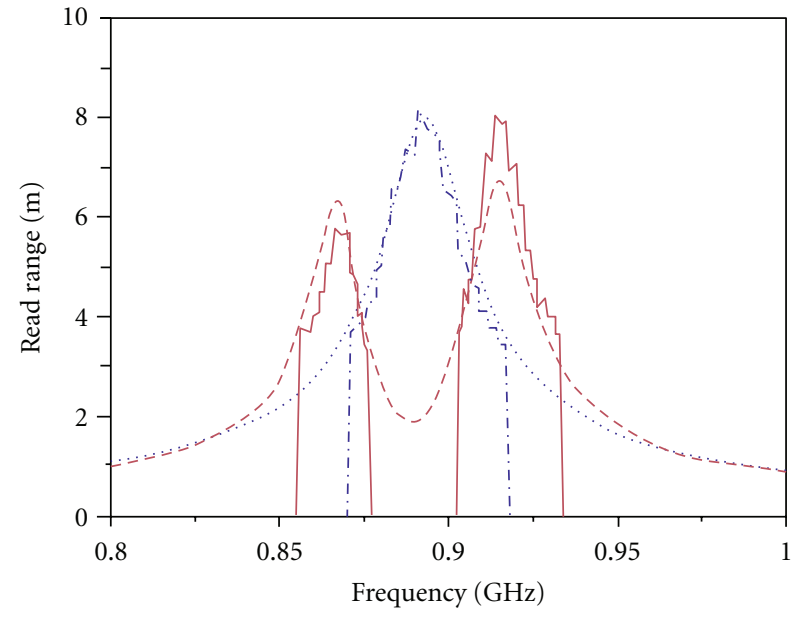

Simulation monoband tag -..- Measures monoband tag

- - Simulation dual-band tag — Measures dual-band tag

(a)
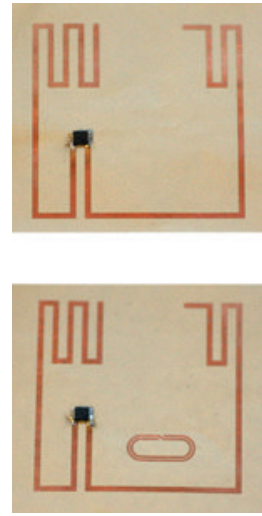

(b)

Figure 31: (a) Simulated and measured read ranges of the monoband and dual-band MLA RFID tags, and (b) fabricated monoband (top) and dual-band (bottom) MLA RFID tags. The considered substrate is the Rogers RO3010 with dielectric constant $\varepsilon_{r}=10.2$ and thickness $h=0.127 \mathrm{~mm}$. From [23]; copyright @2011, IEEE; reprinted with permission.

\section{Applications of Metamaterial Transmission Lines (Part III): Sensors}

Many different sensor types inspired on metamaterial concepts have been reported in the last years (see [150] and references therein). Many of them are based on the split ring resonator (SRR) [24, 151-159], and many others on the CRLH transmission line [160-174], mainly reported by the Group of Professor R. Jakoby at Darmstadt (Germany).
In this paper we report SRR-based microwave sensors, that is, sensors operating in the microwave region of the electromagnetic spectrum. Typically, the operation principle of SRR-based sensors relies on the detuning of one or several resonators due to dielectric loading, strain, displacement, and so forth. For sensing, the change in resonance frequency and/or loss due to detuning is used for determination of the quantity of interest. This principle can be applied to very different sensing applications for mechanical, electrical, 


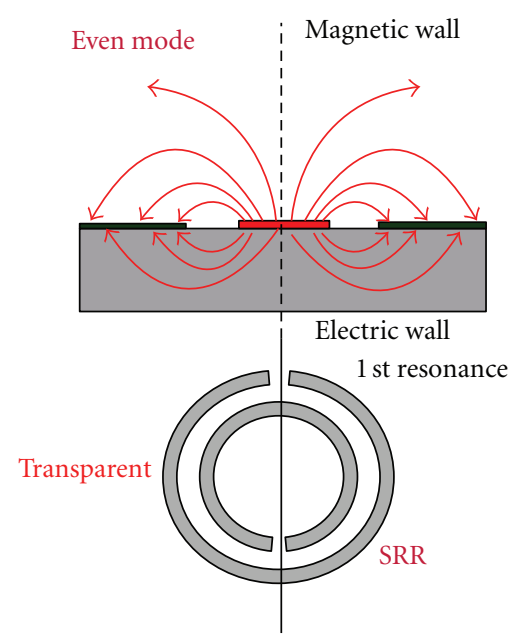

(a)

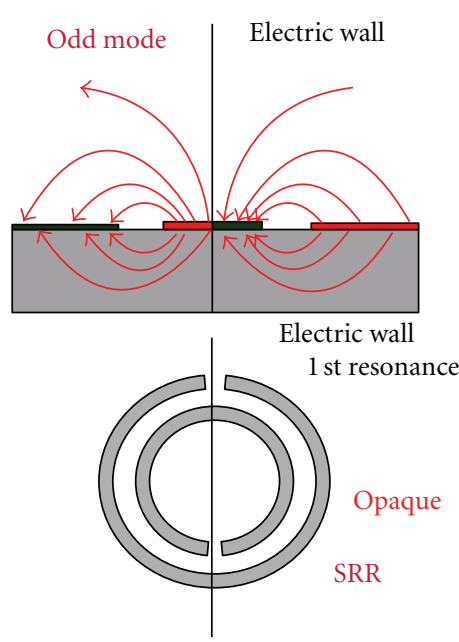

(b)

FIGURE 32: (a) Cross-sectional view of a CPW loaded with a symmetrically etched SRR, and electric field distributions for the fundamental (or even) mode (a) and for the parasitic slot mode (b). For the odd mode the CPW structure exhibits an electric wall in the symmetry plane and it is expected that the SRR causes a notch for this mode if it is symmetrically etched.

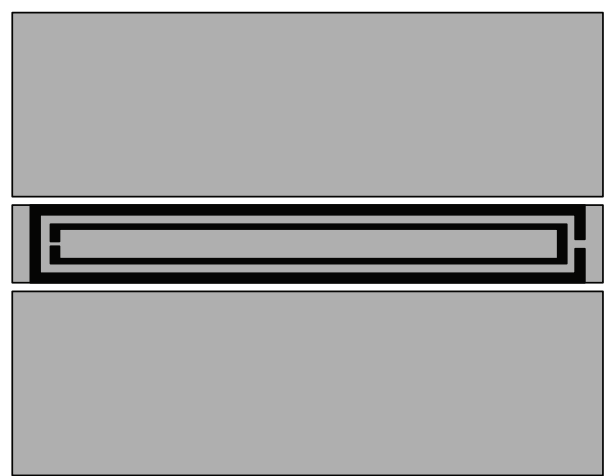

(a)

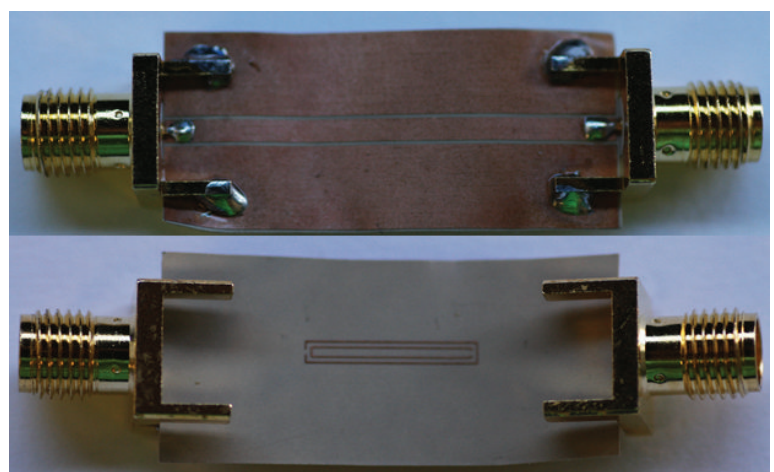

(b)

Figure 33: Layout (a) and photograph (b) of a CPW transmission line loaded with a symmetric SRR. Dimensions are CPW length $L=$ $12.4 \mathrm{~mm}, \mathrm{CPW}$ strip width $W=1.67 \mathrm{~mm}, \mathrm{CPW}$ slot width $G=0.2 \mathrm{~mm}, c=d=0.2 \mathrm{~mm}$, SRR length $l_{1}=12 \mathrm{~mm}$, and SRR width $l_{2}=1.67 \mathrm{~mm}$. The substrate is the Rogers RO3010, with dielectric constant $\varepsilon_{r}=10.2$, thickness $h=127 \mu \mathrm{m}$, and loss tangent tan $\delta=0.0023$.

or chemical quantities, for instance, strain [156, 168] or displacement [152], dielectric properties of biological substances $[154,155]$, and the detection of molecules [158], among others.

In this paper we report SRR-based sensors, but rather than based on detuning, such sensors rely on the symmetry properties of SRRs or other metamaterial resonators. Let us consider for instance a CPW transmission line loaded with a symmetrically etched single SRR (Figure 32 ). With this configuration, due to symmetry, there is not a net axial magnetic field in the SRR (for the fundamental or even mode), this particle is not excited, and the line is transparent. The symmetry plane of the CPW transmission line is a magnetic wall for the fundamental mode, whereas the SRR exhibits an electric wall at this plane if it is symmetrically etched in the CPW. This is an alternative explanation of the transparency of the symmetric SRRloaded CPW. However, by rotating or laterally displacing the resonator, symmetry is truncated, and a notch in the transmission coefficient appears. The magnitude of this notch depends on the deviation from symmetry and hence we can detect displacement or rotation. An advantage of this configuration is that the sensor is insensitive to variations in ambient conditions, since the sensing variable is not the resonator frequency. Based on this principle, prototype sensors have been developed (Figure 33) [24]. Figure 34 depicts the variation of the notch magnitude with lateral displacement and rotations of the SRR. As can be seen, significant sensitivity is achieved. Hence, these symmetric structures are useful for sensing and are of special interest for displacement and rotation sensors, alignment sensors, and so forth. 


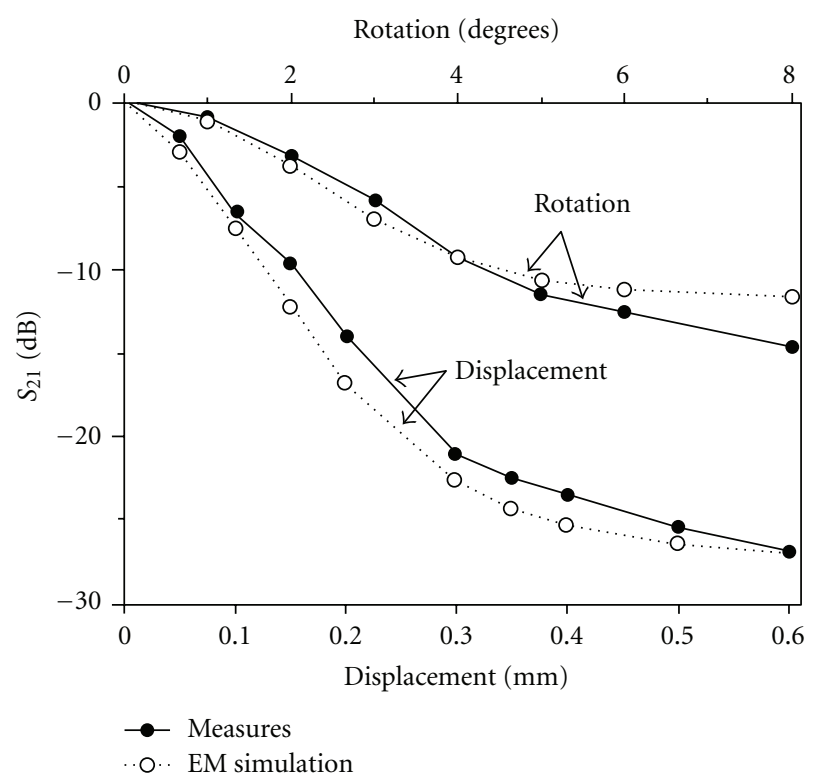

FIgURE 34: Insertion loss at first resonance for the prototype device shown in Figure 33, resulting by laterally displacing and by rotating the SRR. From [24]; copyright (C) 2011, MDPI Publishing; reprinted with permission.

The symmetry properties of transmission lines loaded with symmetric resonators have also been applied to the design of differential (balanced) lines with common mode suppression [175], and to the design of radiofrequency bar codes [149].

\section{Conclusions}

In summary, it has been shown that metamaterial transmission lines, consisting of host lines loaded with reactive elements, exhibit further design flexibility as compared to conventional lines. In these artificial lines, both the characteristic impedance and the dispersion can be engineered or tailored, and many advantages are derived from this fact. Indeed, metamaterial transmission lines have opened the path to a novel design approach of planar microwave circuits based on dispersion and impedance engineering. Compact size, high performance, and novel functional devices are some of the benefits of metamaterial transmission lines. In this paper, it has been shown that these lines can be applied to the design of broadband filters, multiband components, dual-band tags for UHF-RFID, and sensors based on the symmetry properties of SRR-loaded lines. However, many other applications have been demonstrated, and others are expected in the near future.

\section{Acknowledgments}

This work has been supported by Spain-MICIIN (Project Contracts TEC2010-17512 METATRANSFER and TEC201113615-E). Thanks are also given to the Catalan Government for funding CIMITEC and for giving support through the Project 2009SGR-421. This work has also been supported through the CONSOLIDER-INGENIO 2010 Program (Spain-MICIIN) under Project no. CSD2008-00066.

\section{References}

[1] G. V. Eleftheriades and K. G. Balmain, Eds., NegativeRefraction Metamaterials: Fundamental Principles and Applications, John Wiley \& Sons, New York, NY, USA, 2005.

[2] C. Caloz and T. Itoh, Electromagnetic Metamaterials: Transmission Line Theory and Microwave Applications, John Wiley \& Sons, New York, NY, USA, 2006.

[3] N. Engheta and R. W. Ziolkowski, Metamaterials: Physics and Engineering Explorations, John Wiley \& Sons, New York, NY, USA, 2006.

[4] A. K. Sarychev and V. M. Shalaev, Electrodynamics of Metamaterials, World Scientific Publishing, Singapore, 2007.

[5] P. Markos and C. M. Sokoulis, Wave Propagation: From Electrons to Photonic Crystals and Left- Handed Materials, Princeton University Press, 2008.

[6] R. Marques, F. Martín, and M. Sorolla, Metamaterials with Negative Parameters: Theory, Design and Microwave Applications, John Wiley \& Sons, New York, NY, USA, 2008.

[7] F. Capolino, Ed., Metamaterials Handbook, CRC Press, New York, NY, USA, 2009.

[8] L. Solymar and E. Shamonina, Waves in Metamaterials, Oxford University Press, Oxford, UK, 2009.

[9] T. J. Cui and D. R. Smith, Metamaterials: Theory, Design and Applications, Springer, New York, NY, USA, 2010.

[10] R. A. Shelby, D. R. Smith, and S. Schultz, "Experimental verification of a negative index of refraction," Science, vol. 292, no. 5514, pp. 77-79, 2001.

[11] J. B. Pendry, A. J. Holden, D. J. Robbins, and W. J. Stewart, "Magnetism from conductors and enhanced nonlinear phenomena," IEEE Transactions on Microwave Theory and Techniques, vol. 47, no. 11, pp. 2075-2084, 1999.

[12] D. Schurig, J. J. Mock, B. J. Justice et al., "Metamaterial electromagnetic cloak at microwave frequencies," Science, vol. 314, no. 5801, pp. 977-980, 2006.

[13] D. R. Smith, W. J. Padilla, D. C. Vier, S. C. Nemat-Nasser, and S. Schultz, "Composite medium with simultaneously negative permeability and permittivity," Physical Review Letters, vol. 84, no. 18, pp. 4184-4187, 2000.

[14] A. Grbic and G. V. Eleftheriades, "Experimental verification of backward-wave radiation from a negative refractive index metamaterial," Journal of Applied Physics, vol. 92, no. 10, pp. 5930-5935, 2002.

[15] M. Gil, J. Bonache, J. Selga, J. García-García, and F. Martín, "Broadband resonant-type metamaterial transmission lines," IEEE Microwave and Wireless Components Letters, vol. 17, no. 2, pp. 97-99, 2007.

[16] M. Durán-Sindreu, A. Vélez, F. Aznar, G. Sisó, J. Bonache, and F. Martín, "Applications of open split ring resonators and open complementary split ring resonators to the synthesis of artificial transmission lines and microwave passive components," IEEE Transactions on Microwave Theory and Techniques, vol. 57, no. 12, pp. 3395-3403, 2009.

[17] J. Bonache, I. Gil, J. García-García, and F. Martín, "Novel microstrip band pass filters based on complementary split rings resonators," IEEE Transactions on Microwave Theory and Techniques, vol. 54, pp. 265-271, 2006. 
[18] M. Gil, J. Bonache, J. García-García, J. Martel, and F. Martín, "Composite right/left-handed metamaterial transmission lines based on complementary split-rings resonators and their applications to very wideband and compact filter design," IEEE Transactions on Microwave Theory and Techniques, vol. 55, no. 6, pp. 1296-1304, 2007.

[19] M. Durán-Sindreu, A. Vélez, G. Sisó et al., "Recent advances in metamaterial transmission lines based on split rings," Proceedings of the IEEE, vol. 99, pp. 1701-1710, 2011.

[20] J. B. Pendry, "Negative refraction makes a perfect lens," Physical Review Letters, vol. 85, no. 18, pp. 3966-3969, 2000.

[21] V. G. Veselago, "The electrodynamics of substances with simultaneously negative values of $\varepsilon$ and $\mu$," Soviet Physics Uspekhi, vol. 10, pp. 509-514, 1968.

[22] M. Duran-Sindreu, G. Siso, J. Bonache, and F. Martin, "Planar multi-band microwave components based on the generalized composite right/left handed transmission line concept," IEEE Transactions on Microwave Theory and Techniques, vol. 58, no. 12, pp. 3882-3891, 2010.

[23] F. Paredes, G. Zamora, F. J. Herraiz-Martínez, F. Martín, and J. Bonache, "Dual-band UHF-RFID tags based on meander line antennas loaded with spiral resonators," IEEE Antennas and Wireless Propagation Letters, vol. 10, pp. 768-771, 2011.

[24] J. Naqui, M. Durán-Sindreu, and F. Martín, "Novel sensors based on the symmetry properties of split ring resonators (SRRs)," Sensors, vol. 11, no. 8, pp. 7545-7553, 2011.

[25] D. M. Pozar, Microwave Engineering, Addison Wesley, 1990.

[26] B. C. Wadell, Transmission Line Design Handbook, Artech House, 1991.

[27] J. C. Freeman, Fundamentals of Microwave Transmission Lines, John Wiley \& Sons, New York, NY, USA, 1996.

[28] I. Bahl and P. Barthia, Microwave Solid State Circuit Design, John Wiley \& Sons, New York, NY, USA, 2nd edition, 2003.

[29] T. C. Edwards and M. B. Steer, Foundations of Interconnect and Microstrip Design, John Wiley, 3rd edition, 2000.

[30] A. K. Iyer and G. V. Eleftheriades, "Negative refractive index metamaterials supporting 2-D waves," in IEEE-MTT International Microwave Symposium, vol. 2, pp. 412-415, Seattle, Wass, USA, June 2002.

[31] A. A. Oliner, "A periodic-structure negative-refractive-index medium without resonant elements," in URSI Digest, IEEEAP-S USNC/URSI National Radio Science Meeting, p. 41, San Antonio, Tex, USA, June 2002.

[32] C. Caloz and T. Itoh, "Application of the transmission line theory of left-handed (LH) materials to the realization of a microstrip 'LH line', in Proceedings of the IEEE Antennas and Propagation Society International Symposium, vol. 2, pp. 412415, San Antonio, Tex, USA, June 2002.

[33] S. Ramo, J. R. Whinnery, and T. Van Duzer, Fields and Waves in Communication Electronics, John Wiley \& Sons, 1994.

[34] J. A. Kong, Electromagnetic Wave Theory, EMW, 2000.

[35] C. Caloz and T. Itoh, "Invited-novel microwave devices and structures based on the transmission line approach of metamaterials," in Proceedings of the IEEE MTT-S International Microwave Symposium Digest, pp. 195-198, Philadelphia, Pa, USA, June 2003.

[36] C. Caloz, "Dual Composite Right/Left-Handed (D-CRLH) transmission line metamaterial," IEEE Microwave and Wireless Components Letters, vol. 16, no. 11, pp. 585-587, 2006.

[37] G. Sisó, M. Gil, J. Bonache, and F. Martin, "Generalized model for multiband metamaterial transmission lines," IEEE Microwave and Wireless Components Letters, vol. 18, no. 11, pp. 728-730, 2008.
[38] A. Rennings, S. Otto, J. Mosig, C. Caloz, and I. Wolff, "Extended Composite Right/Left-Handed (E-CRLH) metamaterial and its application as quadband quarter-wavelength transmission line," in Proceedings of the Asia-Pacific Microwave Conference (APMC '06), pp. 1405-1408, Yokohama, Japan, December 2006.

[39] G. V. Eleftheriades, "A generalized Negative-Refractive-Index Transmission-Line (NRI-TL) metamaterial for dual-band and quad-band applications," IEEE Microwave and Wireless Components Letters, vol. 17, no. 6, pp. 415-417, 2007.

[40] C. Caloz, H. Okabe, T. Iwai, and T. Itoh, "Transmission line approach of left handed materials," in USNC/URSI National Radio Science Meeting, vol. 1, p. 39, San Antonio, Tex, USA, June 2002.

[41] R. Marqués, F. Mesa, J. Martel, and F. Medina, "Comparative analysis of edge- and broadside-coupled split ring resonators for metamaterial design-theory and experiments," IEEE Transactions on Antennas and Propagation, vol. 51, no. $10 \mathrm{I}$, pp. 2572-2581, 2003.

[42] J. García-García, F. Martín, J. D. Baena, R. Marqús, and L. Jelinek, "On the resonances and polarizabilities of split ring resonators," Journal of Applied Physics, vol. 98, no. 3, Article ID 033103, 9 pages, 2005.

[43] J. D. Baena, R. Marqués, F. Medina, and J. Martel, "Artificial magnetic metamaterial design by using spiral resonators," Physical Review B, vol. 69, no. 1, Article ID 014402, 2004.

[44] F. Falcone, F. Martín, J. Bonache et al., "Stop-band and bandpass characteristics in coplanar waveguides coupled to spiral resonators," Microwave and Optical Technology Letters, vol. 42, no. 5, pp. 386-388, 2004.

[45] R. Marqués, F. Medina, and R. Rafii-El-Idrissi, "Role of bianisotropy in negative, permeability and left-handed metamaterials," Physical Review B, vol. 65, no. 14, Article ID 144440, 2002.

[46] M. Shamonin, E. Shamonina, V. Kalinin, and L. Solymar, "Resonant frequencies of A split-ring resonator: analytical solutions and numerical simulations," Microwave and Optical Technology Letters, vol. 44, no. 2, pp. 133-136, 2005.

[47] F. Falcone, T. Lopetegi, J. D. Baena, R. Marqués, F. Martín, and M. Sorolla, "Effective negative- $\varepsilon$ stopband microstrip lines based on complementary split ring resonators," IEEE Microwave and Wireless Components Letters, vol. 14, no. 6, pp. 280-282, 2004.

[48] F. Falcone, T. Lopetegi, M. A. G. Laso et al., "Babinet principle applied to the design of metasurfaces and metamaterials," Physical Review Letters, vol. 93, no. 19, paper 197401, 2004.

[49] J. Martel, R. Marqués, F. Falcone et al., "A new LC series element for compact bandpass filter design," IEEE Microwave and Wireless Components Letters, vol. 14, no. 5, pp. 210-212, 2004.

[50] J. Martel, J. Bonache, R. Marqués, F. Martín, and F. Medina, "Design of wide-band semi-lumped bandpass filters using open split ring resonators," IEEE Microwave and Wireless Components Letters, vol. 17, no. 1, pp. 28-30, 2007.

[51] A. Vélez, F. Aznar, J. Bonache, M. C. Velázquez-Ahumada, J. Martel, and F. Martín, "Open Complementary Split Ring Resonators (OCSRRs) and their application to wideband cpw band pass filters," IEEE Microwave and Wireless Components Letters, vol. 19, no. 4, pp. 197-199, 2009.

[52] F. Martín, F. Falcone, J. Bonache, R. Marqués, and M. Sorolla, "Miniaturized coplanar waveguide stop band filters based on multiple tuned split ring resonators," IEEE Microwave and Wireless Components Letters, vol. 13, no. 12, pp. 511-513, 2003. 
[53] F. Falcone, F. Martin, J. Bonache, R. Marqués, and M. Sorolla, "Coplanar waveguide structures loaded with splitring resonators," Microwave and Optical Technology Letters, vol. 40, no. 1, pp. 3-6, 2004.

[54] J. García-García, J. Bonache, F. Falcone et al., "Steppedimpedance lowpass filters with spurious passband suppression," Electronics Letters, vol. 40, no. 14, pp. 881-883, 2004.

[55] J. García-García, F. Martín, F. Falcone et al., "Spurious passband suppression in microstrip coupled line band pass filters by means of split ring resonators," IEEE Microwave and Wireless Components Letters, vol. 14, no. 9, pp. 416-418, 2004.

[56] I. Gil, J. García-García, J. Bonache, F. Martín, M. Sorolla, and R. Marqués, "Varactor-loaded split ring resonators for tunable notch filters at microwave frequencies," Electronics Letters, vol. 40, no. 21, pp. 1347-1348, 2004.

[57] J. García-García, J. Bonache, I. Gil et al., "Comparison of electromagnetic band gap and split-ring resonator microstrip lines as stop band structures," Microwave and Optical Technology Letters, vol. 44, no. 4, pp. 376-379, 2005.

[58] J. García-García, F. Martín, F. Falcone et al., "Microwave filters with improved stopband based on sub-wavelength resonators," IEEE Transactions on Microwave Theory and Techniques, vol. 53, no. 6, pp. 1997-2004, 2005.

[59] F. Martín, J. Bonache, F. Falcone, M. Sorolla, and R. Marqués, "Split ring resonator-based left-handed coplanar waveguide," Applied Physics Letters, vol. 83, no. 22, pp. 4652-4654, 2003.

[60] F. Aznar, J. Bonache, and F. Martín, "Improved circuit model for left-handed lines loaded with split ring resonators," Applied Physics Letters, vol. 92, no. 4, paper 043512, 2008.

[61] J. D. Baena, J. Bonache, F. Martín et al., "Equivalent-circuit models for split-ring resonators and complementary splitring resonators coupled to planar transmission lines," IEEE Transactions on Microwave Theory and Techniques, vol. 53, no. 4, pp. 1451-1460, 2005.

[62] M. Gil, J. Bonache, I. Gil, J. García-García, and F. Martín, "On the transmission properties of left-handed microstrip lines implemented by complementary split rings resonators," International Journal of Numerical Modelling, vol. 19, no. 2, pp. 87-103, 2006.

[63] J. Bonache, M. Gil, O. García-Abad, and F. Martín, "Parametric analysis of microstrip lines loaded with complementary split ring resonators," Microwave and Optical Technology Letters, vol. 50, no. 8, pp. 2093-2096, 2008.

[64] A. C. Papanastasiou, G. E. Georghiou, and G. V. Eleftheriades, "A quad-band wilkinson power divider using generalized NRI transmission lines," IEEE Microwave and Wireless Components Letters, vol. 18, no. 8, pp. 521-523, 2008.

[65] M. Studniberg and G. V. Eleftheriades, "A dual-band bandpass filter based on generalized negative-refractive-index transmission-lines," IEEE Microwave and Wireless Components Letters, vol. 19, no. 1, pp. 18-20, 2009.

[66] H. Okabe, C. Caloz, and T. Itoh, "A compact enhancedbandwidth hybrid ring using an artificial lumped-element left-handed transmission-line section," IEEE Transactions on Microwave Theory and Techniques, vol. 52, no. 3, pp. 798-804, 2004.

[67] M. A. Antoniades and G. V. Eleftheriades, "A broadband series power divider using zero-degree metamaterial phaseshifting lines," IEEE Microwave and Wireless Components Letters, vol. 15, no. 11, pp. 808-810, 2005.

[68] G. Sisó, M. Gil, J. Bonache, and F. Martín, "Application of metamaterial transmission lines to design of quadrature phase shifters," Electronics Letters, vol. 43, no. 20, pp. 10981100, 2007.
[69] D. Kholodnyak, E. Serebryakova, I. Vendik, and O. Vendik, "Broadband digital phase shifter based on switchable rightand left-handed transmission line sections," IEEE Microwave and Wireless Components Letters, vol. 16, no. 5, pp. 258-260, 2006.

[70] O. G. Vendik, I. B. Vendik, D. V. Kholodnyak, S. P. Zubko, and E. V. Serebryakova, "Electronically controlled phase shifters based on right/left-handed transmission lines," in Proceedings of the European Microwave Conference, vol. 2, pp. 909-912, Paris, France, October 2005.

[71] M. A. Antoniades and G. V. Eleftheriades, "A broadband Wilkinson balun using microstrip metamaterial lines," IEEE Antennas and Wireless Propagation Letters, vol. 4, no. 1, pp. 209-212, 2005.

[72] G. Sisó, J. Bonache, M. Gil, J. García-García, and F. Martín, "Compact rat-race hybrid coupler implemented through artificial left handed and right handed lines," in Proceedings of the IEEE MTT-S International Microwave Symposium (IMS '07), pp. 25-28, Honolulu, Hawaii, USA, June 2007.

[73] G. Sisó, M. Gil, J. Bonache, and F. Martín, "Applications of resonant-type metamaterial transmission lines to the design of enhanced bandwidth components with compact dimensions," Microwave and Optical Technology Letters, vol. 50, no. 1, pp. 127-134, 2008.

[74] I.-H. Lin, M. DeVincentis, C. Caloz, and T. Itoh, "Arbitrary dual-band components using composite right/left-handed transmission lines," IEEE Transactions on Microwave Theory and Techniques, vol. 52, no. 4, pp. 1142-1149, 2004.

[75] I.-H. Lin, K. M. K. H. Leong, C. Caloz, and T. Itoh, "Dual-band sub-harmonic quadrature mixer using composite right/left-handed transmission lines," IEE Proceedings: Microwaves, Antennas and Propagation, vol. 153, no. 4, pp. 365-375, 2006.

[76] C.-H. Tseng and T. Itoh, "Dual-band bandpass and bandstop filters using composite right/left-handed metamaterial transmission lines," in Proceedings of the IEEE MTT-S International Microwave Symposium Digest, pp. 931-934, San Francisco, Calif, USA, June 2006.

[77] S. H. Ji, O. S. Cho, J. W. Lee, and J. Kim, "Concurrent dualband class-E power amplifier using composite right/lefthanded transmission lines," IEEE Transactions on Microwave Theory and Techniques, vol. 55, no. 6, pp. 1341-1347, 2007.

[78] X. Q. Lin, R. P. Liu, X. M. Yang et al., "Arbitrarily dualband components using simplified structures of conventional CRLH TLs," IEEE Transactions on Microwave Theory and Techniques, vol. 54, no. 7, pp. 2902-2908, 2006.

[79] J. Bonache, G. Sisó, M. Gil, A. Iniesta, J. García-Rincón, and F. Martin, "Application of composite right/left handed (CRLH) transmission lines based on complementary split ring resonators (CSRRs) to the design of dual-band microwave components," IEEE Microwave and Wireless Components Letters, vol. 18, no. 8, pp. 524-526, 2008.

[80] G. Sisó, J. Bonache, and F. Martín, "Dual-band Y-junction power dividers implemented through artificial lines based on complementary resonators," in Proceedings of the IEEE MTTS International Microwave Symposium Digest (MTT '08), pp. 663-666, Atlanta, Ga, USA, June 2008.

[81] L. Liu, C. Caloz, C. C. Chang, and T. Itoh, "Forward coupling phenomena between artificial left-handed transmission lines," Journal of Applied Physics, vol. 92, no. 9, pp. 55605565, 2002.

[82] C. Caloz and T. Itoh, "A novel mixed conventional microstrip and composite right/left-handed backward-wave directional coupler with broadband and tight coupling characteristics," 
IEEE Microwave and Wireless Components Letters, vol. 14, no. 1, pp. 31-33, 2004.

[83] R. Islam, F. Elek, and G. V. Eleftheriades, "Coupledline metamaterial coupler having co-directional phase but contra-directional power flow," Electronics Letters, vol. 40, no. 5, pp. 315-317, 2004.

[84] C. Caloz, A. Sanada, and T. Itoh, "A novel composite right/left-handed coupled-line directional coupler with arbitrary coupling level and broad bandwidth," IEEE Transactions on Microwave Theory and Techniques, vol. 52, no. 3, pp. 980-992, 2004.

[85] E. Jarauta, M. A. G. Laso, T. Lopetegi et al., "Novel microstrip backward coupler with metamaterial cells for fully planar fabrication techniques," Microwave and Optical Technology Letters, vol. 48, no. 6, pp. 1205-1209, 2006.

[86] A. Sanada, C. Caloz, and T. Itoh, "Zeroth-order resonance in composite right/left-handed transmission line resonators," in Asia Pacific Microwave Conference, vol. 3, pp. 1588-1592, Seoul, Korea, 2003.

[87] F. Falcone, F. Martín, J. Bonache, R. Marqués, T. Lopetegi, and M. Sorolla, "Left handed coplanar waveguide band pass filters based on bi-layer split ring resonators," IEEE Microwave and Wireless Components Letters, vol. 14, no. 1, pp. 10-12, 2004

[88] J. Bonache, F. Martín, F. Falcone et al., "Compact coplanar waveguide band-pass filter at the s-band," Microwave and Optical Technology Letters, vol. 46, no. 1, pp. 33-35, 2005.

[89] N. Ortiz, J. D. Baena, M. Beruete et al., "Complementary split-ring resonator for compact waveguide filter design," Microwave and Optical Technology Letters, vol. 46, no. 1, pp. 88-92, 2005.

[90] J. Bonache, I. Gil, J. García-García, and F. Martín, "Complementary split ring resonators for microstrip diplexer design," Electronics Letters, vol. 41, no. 14, pp. 810-811, 2005.

[91] J. Bonache, F. Martin, J. Garcia-Garcia, I. Gil, R. Marqués, and M. Sorolla, "Ultra wide band pass filters (UWBPF) based on complementary split rings resonators," Microwave and Optical Technology Letters, vol. 46, no. 3, pp. 283-286, 2005.

[92] J. Bonache, F. Martín, F. Falcone et al., "Application of complementary split-ring resonators to the design of compact narrow band-pass structures in microstrip technology," Microwave and Optical Technology Letters, vol. 46, no. 5, pp. 508-512, 2005.

[93] J. García-García, J. Bonache, I. Gil, F. Martín, M. Del Castillo Velázquez-Ahumada, and J. Martel, "Miniaturized microstrip and CPW filters using coupled metamaterial resonators," IEEE Transactions on Microwave Theory and Techniques, vol. 54, no. 6, pp. 2628-2635, 2006.

[94] J. Bonache, G. Posada, G. Garchon, W. De Raedt, and F. Martín, "Compact $\left(<0.5 \mathrm{~mm}^{2}\right) \mathrm{K}$-band metamaterial band pass filter in MCM-D technology," Electronics Letters, vol. 43, pp. 45-46, 2007.

[95] M. Gil, J. Bonache, J. García-García, and F. Martín, "Metamaterial filters with attenuation poles in the pass band for ultra wide band (UWB) applications," Microwave and Optical Technology Letters, vol. 49, pp. 2909-2913, 2007.

[96] I. Gil, F. Martin, X. Rottenberg, and W. De Raedt, "Tunable stop-band filter at Q-band based on RF-MEMS metamaterials," Electronics Letters, vol. 43, no. 21, pp. 1153-1154, 2007.

[97] M. Gil, J. Bonache, and F. Martín, "Metamaterial filters: a review," Metamaterials, vol. 2, no. 4, pp. 186-197, 2008.

[98] F. Aznar, A. Velez, M. Duran-Sindreu, J. Bonache, and F. Martin, "Elliptic-function CPW low-pass filters implemented by means of open complementary split ring resonators
(OCSRRs)," IEEE Microwave and Wireless Components Letters, vol. 19, no. 11, pp. 689-691, 2009.

[99] A. Vélez, F. Aznar, M. Durán-Sindreu, J. Bonache, and F. Martín, "Stop-band and band-pass filters in coplanar waveguide technology implemented by means of electrically small metamaterial-inspired open resonators," IET Microwaves, Antennas and Propagation, vol. 4, no. 6, pp. 712-716, 2010.

[100] A. Vélez, G. Sisó, A. Campo, M. Durán-Sindreu, J. Bonache, and F. Martín, "Dual-band microwave duplexer based on spiral resonators (SR) and complementary split ring resonators (CSRR)," Applied Physics A, vol. 103, no. 3, pp. 911-914, 2011.

[101] D. Bouyge, D. Mardivirin, J. Bonache et al., "Split ring resonators (SRRs) based on micro-electro-mechanical deflectable cantilever-type rings: application to tunable stopband filters," IEEE Microwave and Wireless Components Letters, vol. 21, no. 5, pp. 243-245, 2011.

[102] M. Durán-Sindreu, P. Vélez, J. Bonache, and F. Martín, "High-order coplanar waveguide (CPW) filters implemented by means of open split ring resonators (OSRRs) and open complementary split ring resonators (OCSRRs)," Metamaterials, vol. 5, no. 2-3, pp. 51-55, 2011.

[103] M. Durán-Sindreu, P. Vélez, J. Bonache, and F. Martín, "Broadband microwave filters based on open split ring resonators (OSRRs) and open complementary split ring resonators (OCSRRs): improved models and design optimization," Radioengineering, vol. 20, pp. 775-783, 2011.

[104] A. Vélez, P. Vélez, J. Bonache, and F. Martín, "Compact power dividers with filtering capability for ground penetrating radar (GPR) applications," Microwave and Optical Technology Letters, vol. 54, pp. 608-611, 2012.

[105] L. Liu, C. Caloz, and T. Itoh, "Dominant mode leakywave antenna with backfire-to-endfire scanning capability," Electronics Letters, vol. 38, no. 23, pp. 1414-1416, 2002.

[106] S. Lim, C. Caloz, and T. Itoh, "Metamaterial-based electronically controlled transmission-line structure as a novel leakywave antenna with tunable radiation angle and beamwidth," IEEE Transactions on Microwave Theory and Techniques, vol. 52, no. 12, pp. 2678-2690, 2004.

[107] C. Caloz and T. Itoh, "Metamaterials for high-frequency electronics," Proceedings of the IEEE, vol. 93, no. 10, pp. 17441751, 2005.

[108] A. Lai, C. Caloz, and T. Itoh, "Composite right/left-handed transmission line metamaterials," IEEE Microwave Magazine, vol. 5, no. 3, pp. 34-50, 2004.

[109] S. Lim, C. Caloz, and T. Itoh, "A reflecto-directive system using a composite right/left-handed (CRLH) leaky-wave antenna and heterodyne mixing," IEEE Microwave and Wireless Components Letters, vol. 14, no. 4, pp. 183-185, 2004.

[110] C. Caloz and T. Itoh, "Array factor approach of leaky-wave antennas and application to 1-D/2-D composite right/lefthanded (CRLH) structures," IEEE Microwave and Wireless Components Letters, vol. 14, no. 6, pp. 274-276, 2004.

[111] S. Lim, C. Caloz, and T. Itoh, "Electronically scanned composite right/left handed microstrip leaky-wave antenna," IEEE Microwave and Wireless Components Letters, vol. 14, no. 6, pp. 277-279, 2004.

[112] I. Arnedo, J. Illescas, M. Flores et al., "Forward and backward leaky wave radiation in split ring resonator based metamaterials," IET Microwaves, Antennas and Propagation, vol. 1, pp. 65-68, 2007.

[113] N. Engheta and R. W. Ziolkowski, "A positive future for double-negative metamaterials," IEEE Transactions on 
Microwave Theory and Techniques, vol. 53, no. 4, pp. 15351555, 2005.

[114] R. W. Ziolkowski and A. D. Kipple, "Application of Double Negative Materials to Increase the Power Radiated by Electrically Small Antennas," IEEE Transactions on Antennas and Propagation, vol. 51, no. 10, pp. 2626-2640, 2003.

[115] A. Alu and N. Engheta, "Radiation from a travelingwave current sheet at the interface between a conventional material and a metamaterial with negative permittivity and permeability," Microwave and Optical Technology Letters, vol. 35, no. 6, pp. 460-463, 2002.

[116] J. Sun, W. Sun, T. Jiang, and Y. Feng, "Directive electromagnetic radiation of a line source scattered by a conducting cylinder coated with left-handed metamaterial," Microwave and Optical Technology Letters, vol. 47, no. 3, pp. 274-279, 2005.

[117] S. F. Mahmoud, "A new miniaturized annular ring patch resonator partially loaded by a metamaterial ring with negative permeability and permittivity," IEEE Antennas and Wireless Propagation Letters, vol. 3, no. 1, pp. 19-22, 2004.

[118] S. N. Burokur, M. Latrach, and S. Toutain, "Theoretical investigation of a circular patch antenna in the presence of a left-handed medium," IEEE Antennas and Wireless Propagation Letters, vol. 4, no. 1, pp. 183-186, 2005.

[119] M. Kärkkäinen and P. Ikonen, "Patch antenna with stacked split-ring resonators as an artificial magneto-dielectric substrate," Microwave and Optical Technology Letters, vol. 46, no. 6, pp. 544-556, 2005.

[120] F. J. Herraiz-Martínez, L. E. García-Muñoz, D. GonzálezOvejero, V. González-Posadas, and D. Segovia-Vargas, "Dualfrequency printed dipole loaded with split ring resonators," IEEE Antennas and Wireless Propagation Letters, vol. 8, pp. 137-140, 2009.

[121] D. Segovia-Vargas, F. J. Herraiz-Martínez, E. Ugarte-Muñoz, J. Montero-de-Paz, V. González-Posadas, and L. E. GarcíaMuñoz, "Multifrequency printed antennas loaded with metamaterial particles," Radioengineering, vol. 18, no. 2, pp. 129143, 2009.

[122] J. Montero-de-Paz, E. Ugarte-Muñoz, F. J. Herraiz-Martínez, V. Gonzáalez-Posadas, L. E. García-Muñoz, and D. SegoviaVargas, "Multifrequency self-diplexed single patch antennas loaded with split ring resonators," Progress in Electromagnetics Research, vol. 113, pp. 47-66, 2011.

[123] J. Zhu and G. V. Eleftheriades, "Dual-band metamaterialinspired small monopole antenna for WiFi applications," Electronics Letters, vol. 45, no. 22, pp. 1104-1106, 2009.

[124] J. Zhu, M. A. Antoniades, and G. V. Eleftheriades, "A compact tri-band monopole antenna with single-cell metamaterial loading," IEEE Transactions on Antennas and Propagation, vol. 58, no. 4, pp. 1031-1038, 2010.

[125] F. J. Herraiz-Martínez, G. Zamora, F. Paredes, F. Martín, and J. Bonache, "Multiband printed monopole antennas loaded with open complementary split ring resonators for PANs and WLANs," IEEE Antennas and Wireless Propagation Letters, vol. 10, pp. 1528-1531, 2011.

[126] M. Durán-Sindreu, J. Bonache, F. Martín, and T. Itoh, "Novel fully-planar extended-composite right/left handed transmission line based on substrate integrated waveguide for multi-band applications," in European Microwave Conference, Amsterdam, The Netherlands, 2012.

[127] K. Finkenzeller, RFID Handbook, Wiley, New York, NY, USA, 2nd edition, 2003.

[128] B. Glover and H. Bhatt, RFID Essentials, O'Reilly, Sebastopol, Calif, USA, 2006.
[129] D. E. Brown and, RFID Implementation, McGraw-Hill, New York, NY, USA, 2007.

[130] V. D. Hunt, A. Puglia, and M. Puglia, RFID: A Guide to Radiofrequency Identification, Wiley, New York, NY, USA, 2007.

[131] J. Heidrich, D. Brenk, J. Essel et al., "The roots, rules, and rise of RFID," IEEE Microwave Magazine, vol. 11, no. 3, pp. 78-86, 2010.

[132] K. V. S. Rao, P. V. Nikitin, and S. F. Lam, "Antenna design for UHF RFID tags: a review and a practical application," IEEE Transactions on Antennas and Propagation, vol. 53, no. 12, pp. 3870-3876, 2005.

[133] F. Paredes, G. Zamora, J. Bonache, and F. Martín, "Dualband impedance-matching networks based on split-ring resonators for applications in RF identification (RFID)," IEEE Transactions on Microwave Theory and Techniques, vol. 58, no. 5, pp. 1159-1166, 2010.

[134] K. Kurokawa, "Power waves and the scattering matrix," IEEE Transactions on Microwave Theory and Techniques, vol. 13, no. 3, pp. 194-202, 1965.

[135] P. V. Nikitin, K. V. S. Rao, S. F. Lam, V. Pillai, R. Martinez, and H. Heinrich, "Power reflection coefficient analysis for complex impedances in RFID tag design," IEEE Transactions on Microwave Theory and Techniques, vol. 53, no. 9, pp. 27212725, 2005.

[136] M. Hirvonen, N. Pesonen, O. Vermesan, C. Rusu, and P. Enoksson, "Multi-system, multi-band RFID antenna: bridging the gap between HF- and UHF-based RFID applications," in Proceedings of the 38th European Microwave Conference (EuMC '08), pp. 1767-1770, October 2008.

[137] A. Babar, L. Ukkonen, and L. Sydanheimo, "Dual UHF RFID band miniaturized multipurpose planar antenna for compact wireless systems," in Proceedings of the International Workshop on Antenna Technology: Small Antennas, Innovative Structures and Materials (iWAT'10), pp. 1-4, March 2010.

[138] T. Deleruyelle, P. Pannier, E. Bergeret, and S. Bourdel, "Dual band UHF and microwave RFID antenna," in Proceedings of the 38th European Microwave Conference (EuMC '08), pp. 1763-1766, October 2008.

[139] J. Y. Park and J. M. Woo, "Miniaturised dual-band s-shaped RFID tag antenna mountable on metallicsurface," Electronic Letters, vol. 45, pp. 1339-1341, 2008.

[140] Q. Z. Chen and B. J. Hu, "Novel UHF RFID tag antenna with shorted stubs mountable on the metallic objects," in Proceedings of the International Conference on Microwave and Millimeter Wave Technology (ICMMT '08), pp. 1822-1824, April 2008.

[141] S. Aroor and D. D. Deavours, "A dual-resonant microstripbased UHF RFID "cargo" tag," in Proceedings of the IEEE MTT-S International Microwave Symposium Digest (MTT '08), pp. 579-582, June 2008.

[142] G. Zamora, F. Paredes, F. J. Herraiz-Martínez, F. Martín, and J. Bonache, "On the bandwidth limitations ofUHF-RFID tags," to be published.

[143] S. Preradovic and N. C. Karmakar, "Chipless RFID: bar code of the future," IEEE Microwave Magazine, vol. 11, no. 7, pp. 87-97, 2010.

[144] M. Schüßler, C. Damm, M. Maasch, and R. Jakoby, "Performance evaluation of left-handed delay lines for RFID backscatter applications," in Proceedings of the IEEE MTTS International Microwave Symposium Digest (MTT '08), pp. 177-180, June 2008. 
[145] I. Jalaly and I. D. Robertson, "RF bar codes using multiple frequency bands," in Proceedings of the IEEE MTT-S International Microwave Symposium Digest, pp. 4-7, Long Beachm, Calif, USA, June 2005.

[146] J. McVay, A. Hoorfar, and N. Eagheta, "Space-filling curve RFID tags," in Proceedings of the IEEE Radio and Wireless Symposium, pp. 199-202, San Diego, Calif, USA, January 2006.

[147] S. Preradovic, I. Balbin, N. C. Karmakar, and G. F. Swiegers, "Multiresonator-based chipless RFID system for low-cost item tracking," IEEE Transactions on Microwave Theory and Techniques, vol. 57, no. 5, part 2, pp. 1411-1419, 2009.

[148] F. J. Herraiz-Martínez, F. Paredes, G. Zamora Gonzalez, F. Martín, and J. Bonache, "Printed magnetoinductive-wave (MIW) delay lines for chipless RFID applications," IEEE Transactions on Antennas and Propagation, vol. 60, no. 11, pp. 5075-5082, 2012.

[149] J. Naqui, M. Durán-Sindreu, and F. Martín, "On the symmetry properties of coplanar waveguides loaded with symmetric resonators: analysis and potential applications," in IEEE/MTT-S International Microwave Symposium Microwave Symposium, Montreal, Canada, June 2012.

[150] M. Schüßler, C. Mandel, M. Puentes, and R. Jakoby, "Metamaterial inspired microwave sensors," IEEE Microwave Magazine, vol. 13, pp. 57-68, 2012.

[151] E. Ekmekci and G. Turhan-Sayan, "Metamaterial sensor applications based on broadside-coupled SRR and V-shaped resonator structures," in Proceedings of the International Symposium on Antennas and Propagation, pp. 1170-1172, Jeju, South Korea, 2011.

[152] C. Mandel, B. Kubina, M. Schüßler, and R. Jakoby, "Passive chipless wireless sensor for twodimensional displacement measurement," in Proceedings of the 41st European Microwave Conference, pp. 79-82, Manchester, England, 2011.

[153] M. Puentes, C. Weiss, M. Schüßler, and R. Jakoby, "Sensor array based on split ring resonators for analysis of organic tissues," in Proceedings of the IEEE MTT-S International Microwave Symposium Digest, Baltimore, Md, USA, 2011.

[154] H.-J. Lee and J.-G. Yook, "Biosensing using split-ring resonators at microwave regime," Applied Physics Letters, vol. 92, no. 25, Article ID 254103, 2008.

[155] H.-J. Lee, H.-S. Lee, K.-H. Yoo, and J.-G. Yook, "On the possibility of biosensors based on split ring resonators," in Proceedings of the 38th European Microwave Conference, pp. 1222-1225, Amsterdam, The Netherlands, 2008.

[156] R. Melik, E. Unal, N. K. Perkgoz, C. Puttlitz, and H. V. Demir, "Metamaterial-based wireless strain sensors," Applied Physics Letters, vol. 95, no. 1, Article ID 011106, 2009.

[157] T. Driscoll, G. O. Andreev, D. N. Basov et al., "Tuned permeability in terahertz split-ring resonators for devices and sensors," Applied Physics Letters, vol. 91, no. 6, Article ID 062511, 2007.

[158] E. Cubukcu, S. Zhang, Y. S. Park, G. Bartal, and X. Zhang, "Split ring resonator sensors for infrared detection of single molecular monolayers," Applied Physics Letters, vol. 95, no. 4, Article ID 043113, 2009.

[159] J. F. O'Hara, R. Singh, I. Brener et al., "Thin-film sensing with planar terahertz metamaterials: sensitivity and limitations," Optics Express, vol. 16, no. 3, pp. 1786-1795, 2008.

[160] A. Penirschke, M. Schüßler, and R. Jakoby, "New microwave flow sensor based on a left-handed transmission line resonator," in Proceedings of the IEEE MTT-S International
Microwave Symposium (IMS '07), pp. 393-396, Honolulu, Hawaii, USA, June 2007.

[161] A. Angelovski, A. Penirschke, and R. Jakoby, "CRLH-mass flow detector array for cross-sectional detection of inhomogeneous distributed flow regimes in pipelines," in Proceedings of the 41st European Microwave Conference, pp. 611-614, Manchester, England, 2011.

[162] M. Puentes, B. Stelling, M. Schüßler, A. Penirschke, and R. Jakoby, "Planar sensor for permittivity and velocity detection based on metamaterial transmission line resonator," in Proceedings of the 39th European Microwave Conference (EuMC '09), pp. 57-60, Manchester, England, October 2009.

[163] M. Puentes, A. Penirschke, M. Schüßler, and R. Jakoby, "Microwave mass flow meter for industrial applications based on a lefthanded transmission line resonator," in Proceedings of the German Microwave Conference (GeMIC '09), Munich, Germany, March 2009.

[164] C. Damm, M. Schüßler, M. Puentes, H. Maune, M. Maasch, and R. Jakoby, "Artificial transmission lines for high sensitive microwave sensors," in Proceedings of the IEEE Sensors Conference (SENSORS '09), pp. 755-758, Christchurch, New Zealand, October 2009.

[165] M. Sazegar, Y. Zheng, H. Maune et al., "Low-cost phasedarray antenna using compact tunable phase shifters based on ferroelectric ceramics," IEEE Transactions on Microwave Theory and Techniques, vol. 59, no. 5, pp. 1265-1273, 2011.

[166] C. Mandel, H. Maune, M. Maasch, M. Sazegar, M. Schüßler, and R. Jakoby, "Passive wireless temperature sensing with BST-based chipless transponder," in Proceedings of the German Microwave Conference (GeMiC '11), pp. 1101-1104, Darmstadt, Germany, March 2011.

[167] M. Schüßler, M. Maasch, C. Damm, and R. Jakoby, "Compact microstrip patch antennas for passive RFID backscatter tags," in Proceedings of the 39th European Microwave Conference (EuMC '09), pp. 1101-1104, Rome, Italy, October 2009.

[168] C. Mandel, M. Schüßler, and R. Jakoby, "A wireless passive strain sensor," in Proceedings of the IEEE Sensors Conference, pp. 207-210, Limerick, Ireland, 2011.

[169] M. Puentes, B. Stelling, M. Schüßler, A. Penirschke, C. Damm, and R. Jakoby, "Dual mode sensor for belt conveyor systems based on planar metamaterials," in Proceedings of the IEEE Sensors Conference (SENSORS '09), pp. 487-491, Christchurch, New Zealand, October 2009.

[170] M. Schüßler, C. Damm, M. Maasch, and R. Jakoby, "Performance evaluation of left-handed delay lines for RFID backscatter applications," in Proceedings of the IEEE MTTS International Microwave Symposium Digest (MTT '08), pp. 177-180, June 2008.

[171] C. Mandel, M. Schüßler, M. Maasch, and R. Jakoby, "A novel passive phase modulator based on LH delay lines for chipless microwave RFID applications," in Proceedings of the IEEE MTT-S International Microwave Workshop Series on Wireless Sensing, Local Positioning and RFID (IMWS '09), Cavtat, Croatia, September 2009.

[172] M. Puentes, M. Schüßler, C. Damm, and R. Jakoby, "Extraction of capacitive profiles with a planar metamaterial sensor," Applied Physics A, vol. 103, no. 3, pp. 815-819, 2011.

[173] M. Schüßler, M. Puentes, C. Mandel, and R. Jakoby, "Multiresonant perturbation method for capacitive sensing with composite right/left-handed transmission lines," in Proceedings of the IEEE MTT-S International Microwave Symposium (MTT'10), pp. 481-484, Anaheim, Calif, USA, May 2010. 
[174] M. Schüßler, C. Mandel, M. Puentes, and R. Jakoby, "Capacitive level monitoring of layered fillings in vessels using composite right/left-handed transmission lines," in Proceedings of the IEEE MTT-S International Microwave Symposium (MTT'10), Baltimore, Md, USA, 2011.

[175] J. Naqui, A. Fernández-Prieto, M. Durán-Sindreu et al., "Split rings-based differential transmission lines with commonmode suppression," in Proceedings of the IEEE MTT-S International Microwave Symposium (MTT '10), Baltimore, Md, USA, June 2011. 

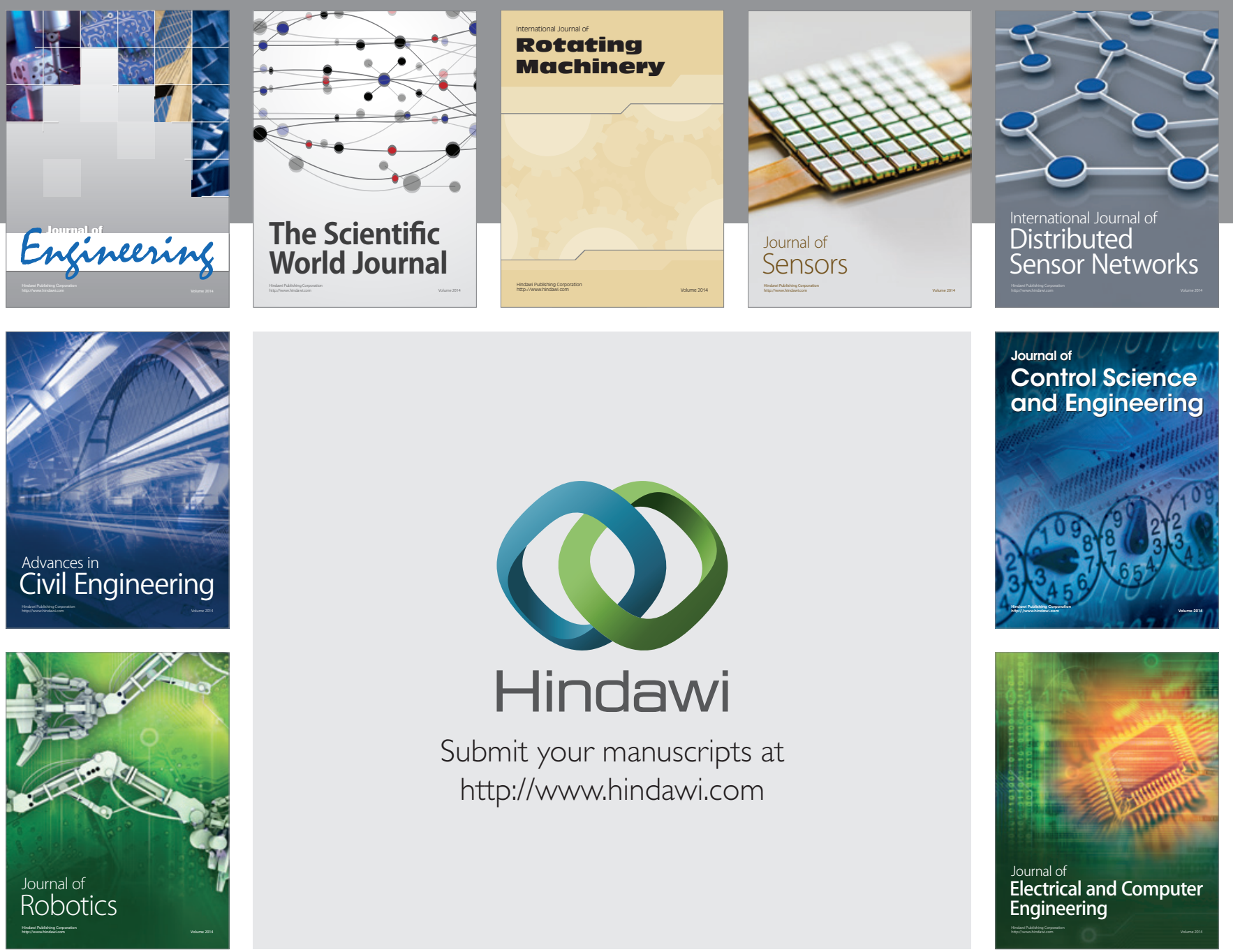

Submit your manuscripts at

http://www.hindawi.com
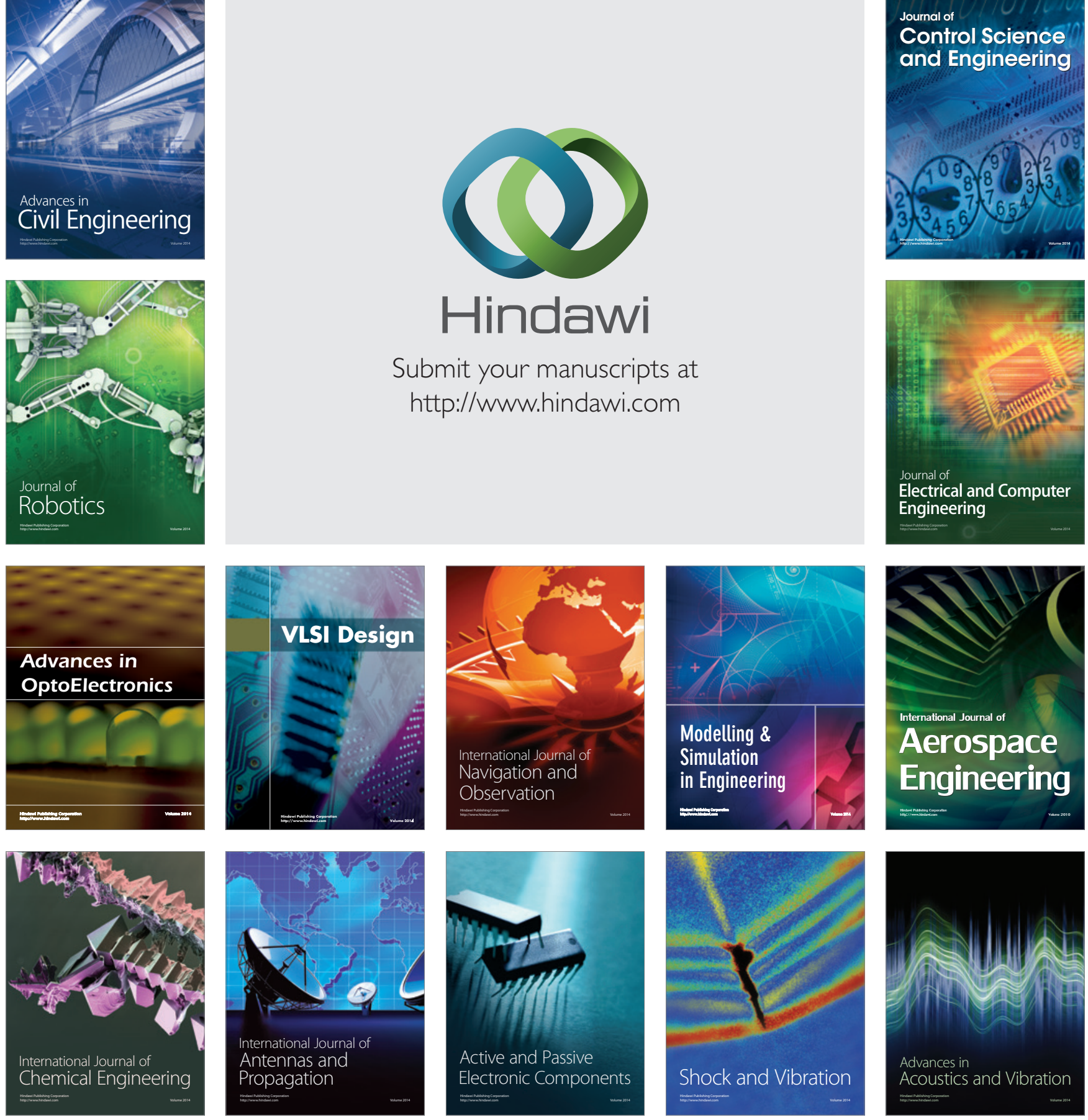